\title{
Differential Incremental Equilibrium Geometry - Spatial Folding of Protein Particles, Genome Expression and Bidirectional Semiconservative Replication of Ring Chromosomes
}

\author{
Zhu Rong Rong \\ Office of Human Resources, Fudan University, Shanghai, China
}

Email address:

rongrongzhu1969@163.com

\section{To cite this article:}

Zhu Rong Rong. Differential Incremental Equilibrium Geometry - Spatial Folding of Protein Particles, Genome Expression and Bidirectional Semiconservative Replication of Ring Chromosomes. American Journal of Applied Mathematics. Special Issue: Molecular Cellular Information Mathematics-Differential Incremental Equilibrium Geometry. Vol. 7, No. 4, 2019, pp. 98-113. doi: 10.11648/j.ajam.20190704.12

Received: July 13, 2019; Accepted: July 31, 2019; Published: September 12, 2019

\begin{abstract}
The research direction of this paper is to study the interdisciplinary subjects of life science, mathematics and computer science at the molecular level from the life science Molecular Cell Biology. On the basis of mathematical primitive innovation "Differential Incremental Balanced Geometry", the cell modification of normal chromosome mitosis was established at the molecular level, and the normal cell tissue spatial morphology with initial boundary was established. DNA is used to unravel double helix and separate double strands to solve the protein skeleton structure of bi-directional Semi-Reserved replication of cyclic chromosomes in life sciences at the molecular level. Therefore, it establishes and reveals the duplication fork and bidirectional duplication of molecular cell biology model, the internal structure and regularity of cyclic chromosomes bound by cyclic DNA double helix and many proteins. New mathematics is integrated into the micro-activities of cell modification in life sciences. The topological geometric image of the solitary wavelet with supersymmetric structure is constructed, which reflects the correct abstract model of cell modification and provides dynamic structure for DNA gene sequencing, etc. It also provides a mature mathematical basis for reliable predictability of gene editing.
\end{abstract}

Keywords: Ring Chromosomes, Chromosome Mitosis, Molecular Cytobiology, Spatial Folding of Protein Particles, Cell Modification, Bidirectional Semi-reserved Replication

\section{Introduction}

\subsection{Topological Mathematics of High Dimensional Fibers and Mitosis}

Chromosome tissue level and chromosome mitosis begin. DNA double helix polypeptide structure presents 8-mer H1 protein, forming nucleosome, nucleosome filament and annular domain. It has a macro-non-holomorphic secondary micro-incremental equilibrium spatial structure of 4-dimensional super-high-end superspherical convex globular fibrous plexus protein chain combination.

\subsection{Cell Modification and Initial Boundary of Topological Space}

The cell modification of normal chromosome mitosis and the spatial morphology of normal cell tissue at the initial boundary form haploid cell space morphology. DNA replication and repair involves protein particle folding and complex polypeptide space unchain, as well as the space structure of DNA unhelix and cell modification and cell division.

\subsection{Chromosome Bidirectional Semi-reserved Replication and 3+1-Dimensional Solitary Wavelet}

DNA unravels double helix and separates double strands, and replicates bi-directionally and Semi-Reserved with circular chromosomes. $3+1$ dimensional dimensionality 
reduction high-end hyperspherical convex spherical fibers cluster-protein particle Space folding and protein particle space construction. Cell modification is the spatial construction and folding correlation of protein particles. It has a complex (core variable) structure of polypeptide spatial deep chain unwinding, forming a single-chain form of protein particles solitary wavelet domain nuclear control similarity solution solution, which can reveal their morphology.

The two duplicate double helixes are separated from each other, and the two duplicate forks move from the same starting point to the opposite direction. When two replication forks meet at the relative points on the ring, the replication terminates.

\subsection{Chromosome Fragment Oric and Protein Spatial Solitary Wavelet}

Duplication fork and bidirectional duplication, cyclic DNA double helix with many proteins binding to the cyclic chromosome. Cyclic DNA double helix binds to many proteins. The starting point of replication is a sequence called oriC. Many proteins bind to it and initiate the replication process. This regulatory sequence serves as a binding site for sequence-specific DNA-binding proteins.

Solitary Wavelet Similarity Solution for the Whole Protein Particles (Peptides) of Myosin $\alpha / \beta$ Bucket (First Order Differential) with 4-Dimensional Super-high-end Superglobular Convex Bundle Fiber Cluster and Protein Chain Combination.

\section{Mathematical Definition and Analysis of Chromosome Mitosis, Bi-directional Semi-reserved Replication and Cell Modification}

\subsection{Folding Conformation and Chain Unwinding of Polypeptide with Initial Boundary}

The microstructures of polypeptides fold into protein particles with weak macrostructure, and the natural conformations constructed by the microstructures of polypeptides have a polypeptide spatial chain-breaking structure with initial boundary.

$$
\begin{array}{r}
a=\int \operatorname{Cos}(z)\left[(-C) \operatorname{Sin}^{2}(b) \cdot \operatorname{Arcctg}\left[\left(2 \operatorname{Cos}^{3}\left(\frac{1}{2} x+\frac{\pi}{4}\right)+2 \operatorname{Sin}\left(y+\frac{\pi}{4}\right)+\operatorname{Sin}\left(\frac{3 \pi}{4}\right)+\operatorname{Sin}^{2}\left(\frac{3 \pi}{4}\right)+\operatorname{Sin}^{3}\left(\frac{3 \pi}{4}\right)\right)\right] d z+\int x \operatorname{Sin}(z) d z+\right. \\
\left.+\operatorname{Sin}(z)\left((-C) \operatorname{Sin}^{2}(b) \cdot \operatorname{Arcctg}\left[\left(2 \operatorname{Cos}^{3}\left(\frac{1}{2} x+\frac{\pi}{4}\right)+2 \operatorname{Sin}\left(y+\frac{\pi}{4}\right)+\operatorname{Sin}\left(\frac{3 \pi}{4}\right)+\operatorname{Sin}^{2}\left(\frac{3 \pi}{4}\right)+\operatorname{Sin}^{3}\left(\frac{3 \pi}{4}\right)\right)\right]\right)+x \operatorname{Cos}(z)\right]
\end{array}
$$

Formula 1. The natural conformational solitary wavelet is constructed by folding the weak microstructures of polypeptides into the space of protein particles with weak macrostructure.

$2+1$ dimension-non-high-end hyperspherical convex spherical fibers cluster-polypeptide weak microstructures fold into protein particles with weak macrostructure to construct a natural conformation of polypeptide spatial unchain structure with initial boundary, forming single-chain protein particles, and spatially transforming $\operatorname{Csc}(y+\pi / 4) \rightarrow \operatorname{Sin}(y+\pi / 4)$ soliton wave-value domain nuclear-controlled similarity solution.

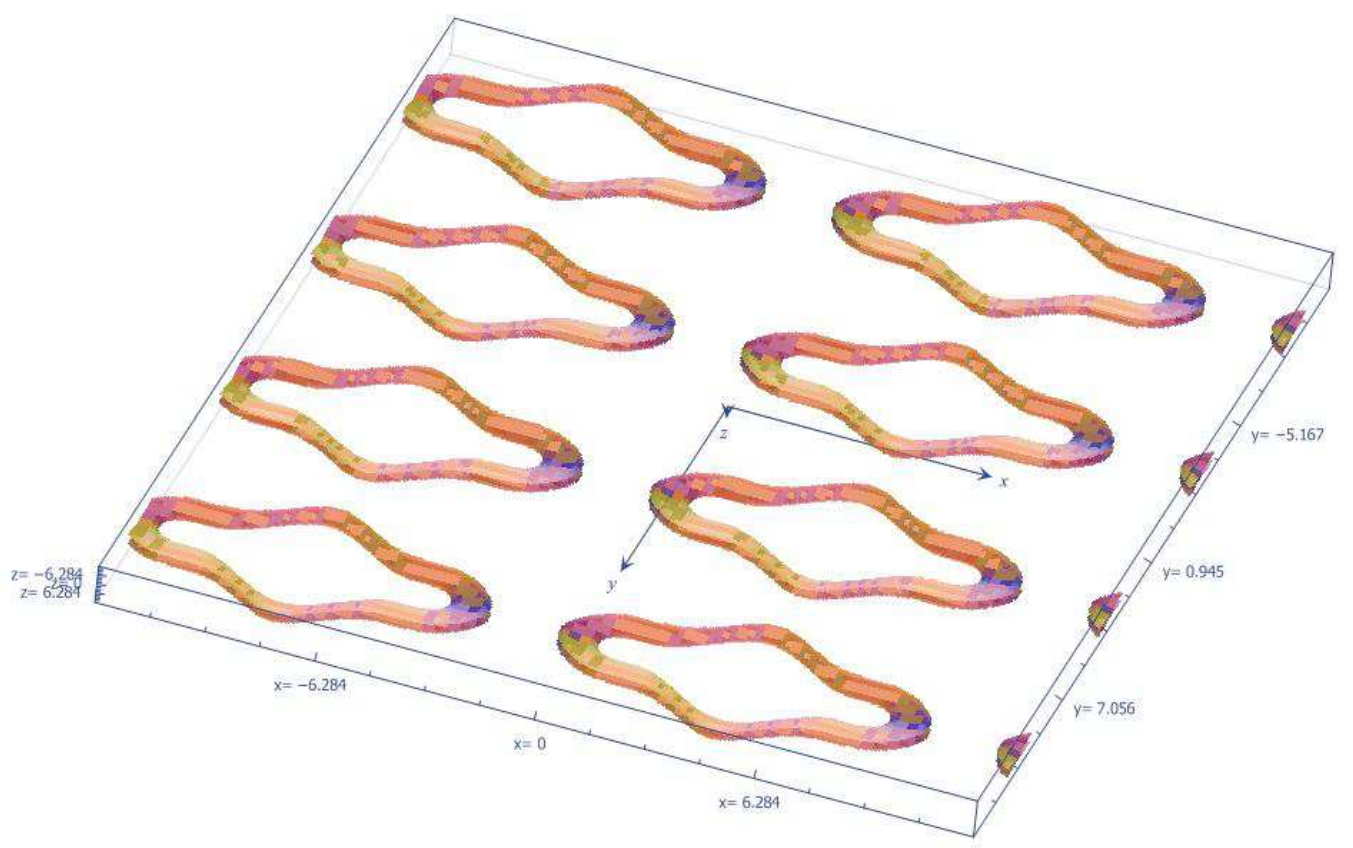

Figure 1. Polypeptide space unchain structure, forming single-stranded protein particles. 


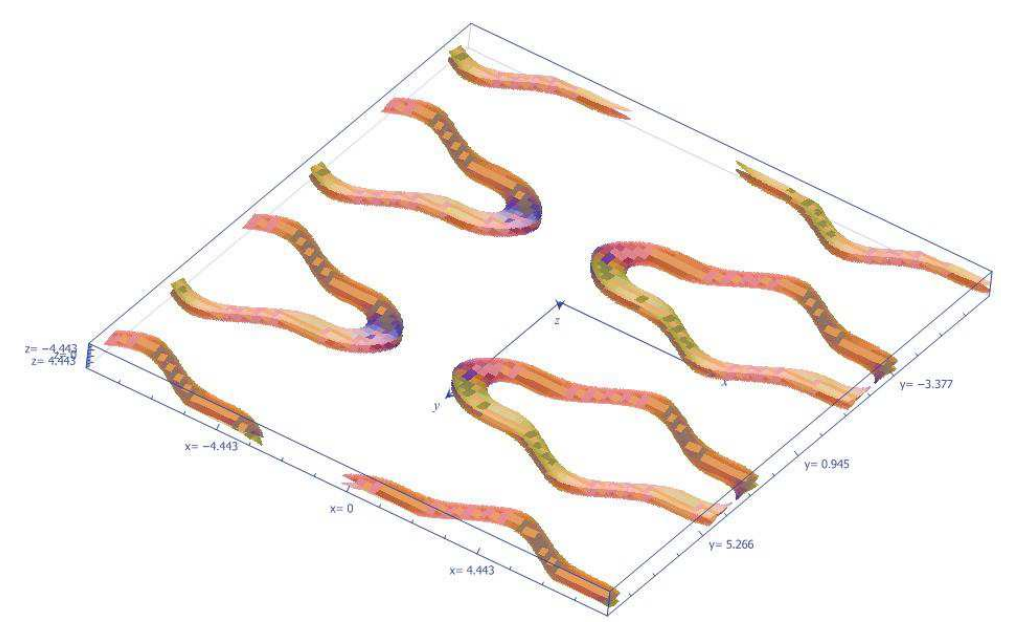

Figure 2. Polypeptide space unchain structure, forming single-stranded protein particles.

\subsection{Peptide Folding Is Associated with H1 Histone, Nucleosome, Annular Domain and Mitotic Cell Modification}

Regulation of nuclear and gene expression and mitosis of chromosomes. Polypeptide folding of protein particles, cyclic domains with H1 histones and nucleosomes, and chromosomes are composed of DNA and its binding proteins. The microstructures of polypeptides fold into protein and cell modification of chromosomal mitosis.

The microstructures of polypeptides are folded into protein

$$
\left\{\begin{array}{l}
r_{(\theta, \varphi)}=\operatorname{ArcSin}\left[\frac{1}{\left.-C \cdot \operatorname{Arcctg}\left[2 \operatorname{Sin}\left(\varphi+\frac{\pi}{4}\right)+\operatorname{Sin}(\theta)+\operatorname{Sin}(\theta) \operatorname{Sin}(\theta)+2 \operatorname{Cos}^{3}\left(\theta+\frac{\pi}{4}\right)\right]\right]}\right] \operatorname{Arc} \operatorname{Cos}\left[\frac{1}{-C \cdot \operatorname{Arctg}\left[2 \operatorname{Sin}\left(\varphi+\frac{\pi}{4}\right)+\operatorname{Sin}(\theta)+\operatorname{Sin}(\theta) \operatorname{Sin}(\theta)+2 \operatorname{Cos}^{3}\left(\theta+\frac{\pi}{4}\right)\right]}\right]+2 \pi N_{1}+\frac{3 \pi}{4} \\
r_{(\theta, \varphi)}=\operatorname{ArcSin}\left[\frac{1}{-C \cdot \operatorname{Arcctg}\left[2 \operatorname{Sin}\left(\varphi+\frac{\pi}{4}\right)+\operatorname{Sin}(\theta)+\operatorname{Sin}(\theta) \operatorname{Sin}(\theta)+2 \operatorname{Cos}^{3}\left(\theta+\frac{\pi}{4}\right)\right]}\right] \operatorname{ArcCos}\left[\frac{1}{-C \cdot \operatorname{Arcctg}\left[2 \operatorname{Sin}\left(\varphi+\frac{\pi}{4}\right)+\operatorname{Sin}(\theta)+\operatorname{Sin}(\theta) \operatorname{Sin}(\theta)+2 \operatorname{Cos}^{3}\left(\theta+\frac{\pi}{4}\right)\right]}\right]+2 \pi N_{2}-\frac{5 \pi}{4}
\end{array}\right.
$$

Formula 2. 4-dimensional super-high-end hyperspherical convex globular fibrous plexus chromosomal tissue hierarchy, and the nucleosome, filament, and cell modification of the particles with weak macrostructure, and the existence of solitary wave similarity solutions for the spatial construction of natural conformations is discussed.

Chromatin tissue layers, naked DNA molecules surround histones to form nucleosomes, the first layer with edge chromatin packaging; nucleosomes form $30 \mathrm{~mm}$ filament (dimension) filament, from $30 \mathrm{~mm}$ filament (dimension) to circular domain. When cells are ready for mitosis, the ring structure is further compressed into mitotic chromosomes.

\section{Polypeptide Space Unchain and Normal Chromosome Polypeptide Folding and 8-Mer H1 Histone}

\subsection{4-dimensional Super-high-end Hyperspherical Convex Spherical Fiber Bundles, 8-mer H1 Protein, Solitary Wavelet} General Solution-Range Nuclear Control

$$
\left\{\begin{array}{l}
r_{(\theta, \varphi)}=\operatorname{ArcSin}\left[\frac{1}{\operatorname{Matrix}\left[-C \cdot \operatorname{Arcctg}\left(\frac{d}{d \varphi} f\left(C,\left(\varphi+\frac{\pi}{4}\right)\right), \frac{d}{d \theta} F\left(C,\left(\theta+\frac{\pi}{4}\right)\right)\right)\right]}\right] \operatorname{ArcCos}\left[\frac{1}{\operatorname{Matrix}\left[-C \cdot \operatorname{Arctg}\left(\frac{d}{d \varphi} f\left(C,\left(\varphi+\frac{\pi}{4}\right)\right), \frac{d}{d \theta} F\left(C,\left(\theta+\frac{\pi}{4}\right)\right)\right)\right]}\right]+2 \pi N_{1}+\frac{3 \pi}{4} \\
r_{(\theta, \varphi)}=\operatorname{ArcSin}\left[\frac{1}{\operatorname{Matrix}\left[-C \cdot \operatorname{Arctg}\left(\frac{d}{d \varphi} f\left(C,\left(\varphi+\frac{\pi}{4}\right)\right), \frac{d}{d \theta} F\left(C,\left(\theta+\frac{\pi}{4}\right)\right)\right)\right]}\right] \operatorname{ArcCos}\left[\frac{1}{\operatorname{Matrix}\left[-C \cdot \operatorname{Arctg}\left(\frac{d}{d \varphi} f\left(C,\left(\varphi+\frac{\pi}{4}\right)\right), \frac{d}{d \theta} F\left(C,\left(\theta+\frac{\pi}{4}\right)\right)\right)\right]}\right]+2 \pi N_{2}-\frac{5 \pi}{4}
\end{array}\right.
$$




$$
\begin{gathered}
\operatorname{Matrix}\left[-C \cdot \operatorname{Arcctg}\left(\frac{d}{d \varphi} f\left(C,\left(\varphi+\frac{\pi}{4}\right)\right), \frac{d}{d \theta} F\left(C,\left(\theta+\frac{\pi}{4}\right)\right)\right)\right]=-C \cdot \operatorname{Arcctg}\left[\frac{d}{d \varphi}\left(2 \operatorname{Sin}\left(\varphi+\frac{\pi}{4}\right)\right)+\frac{d}{d \theta}\left(\operatorname{Sin}\left(\frac{3 \pi}{4}\right)+\operatorname{Sin}\left(\frac{3 \pi}{4}\right) \operatorname{Sin}\left(\frac{3 \pi}{4}\right)+2 \operatorname{Cos}^{3}\left(\theta+\frac{\pi}{4}\right)\right)\right] \\
+\frac{d}{d \theta}\left(\operatorname{Sin}\left(\frac{3 \pi}{4}\right)+\operatorname{Sin}\left(\frac{3 \pi}{4}\right) \operatorname{Sin}\left(\frac{3 \pi}{4}\right)+2 \operatorname{Cos}^{3}\left(\theta+\frac{\pi}{4}\right)\right)
\end{gathered}
$$

Formula 3. 4-dimensional super-high-end superspherical convex spherical fiber bundles, macroscopic non-holomorphic quadratic incremental equilibrium space structure of protein chain combination and binary first-order partial differential equation, and general solution of solitary wave similarity solution of global non-linear partial differential equation system of 8-polymer $\mathrm{H} 1$ protein.

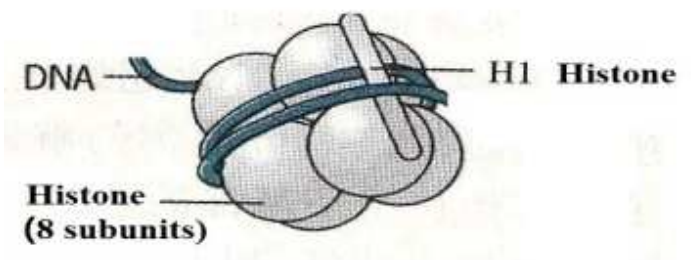

Figure 3. 8 Polymer H1 Protein-Electron Microscope.

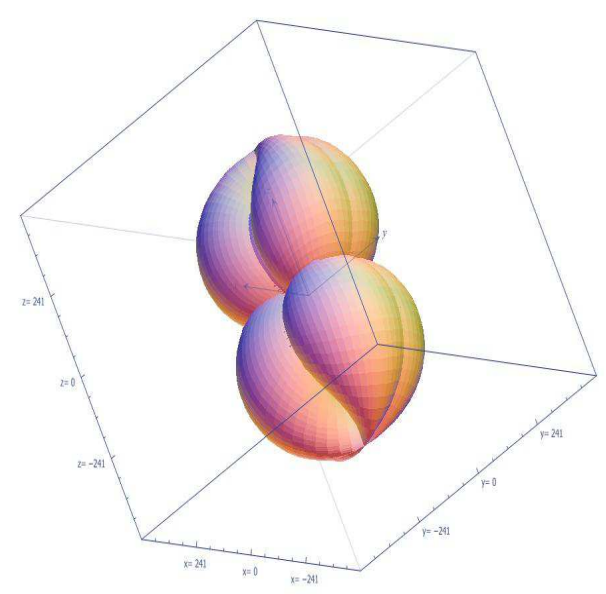

Figure 4. 8 Polymer H1 Protein Mathematical 3D Microscope.

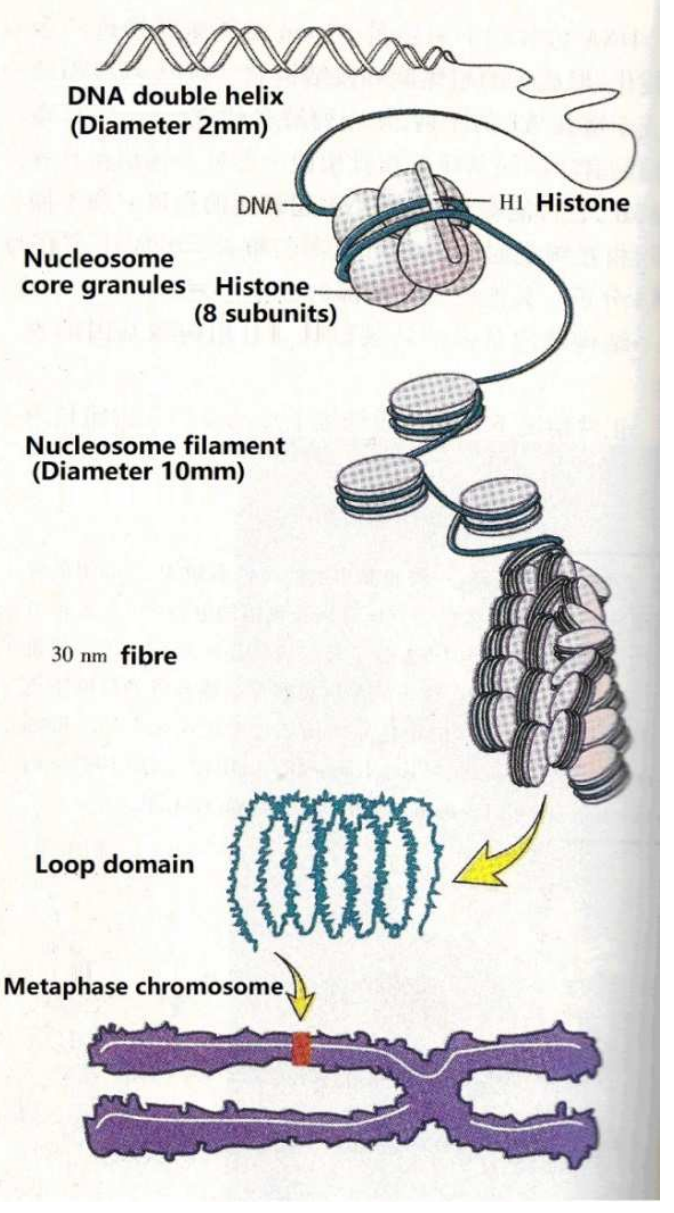

Figure 5. DNA double helix.

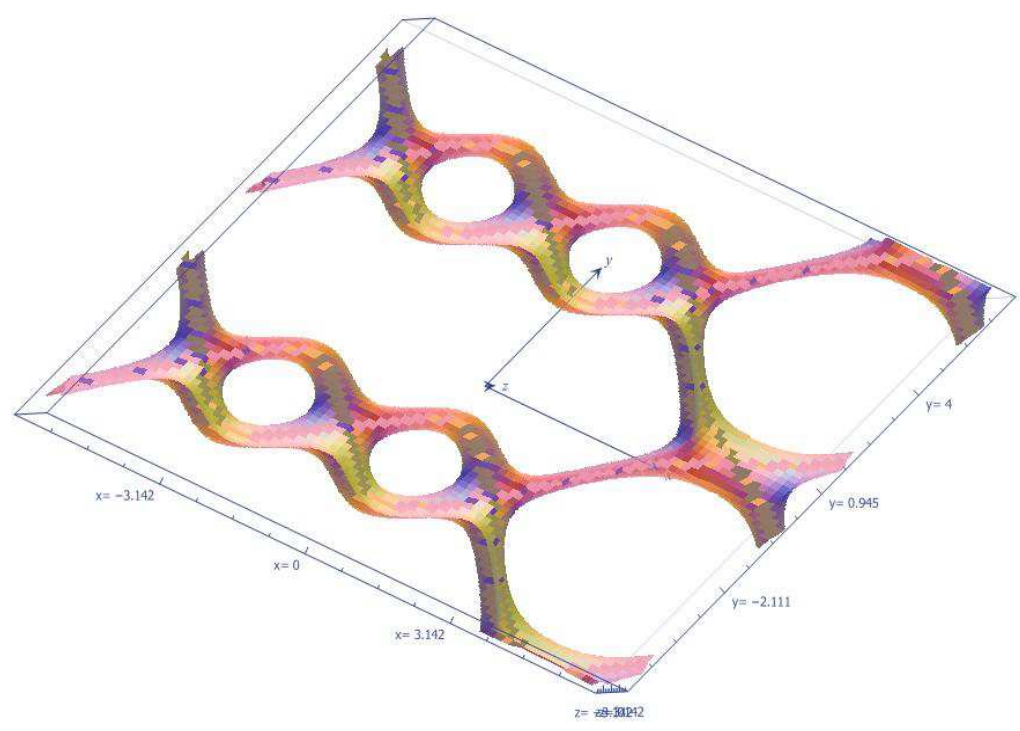

Figure 6. DNA double helix_Polypeptide structure. 


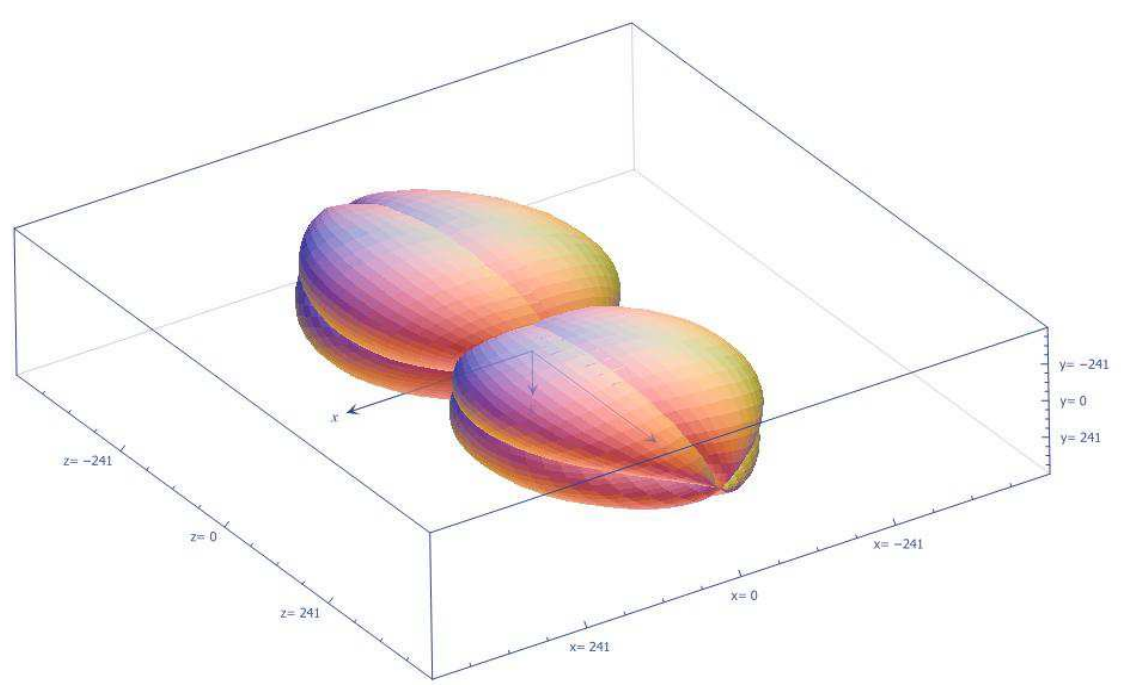

Figure 7. 8-Polymer H1 Protein.

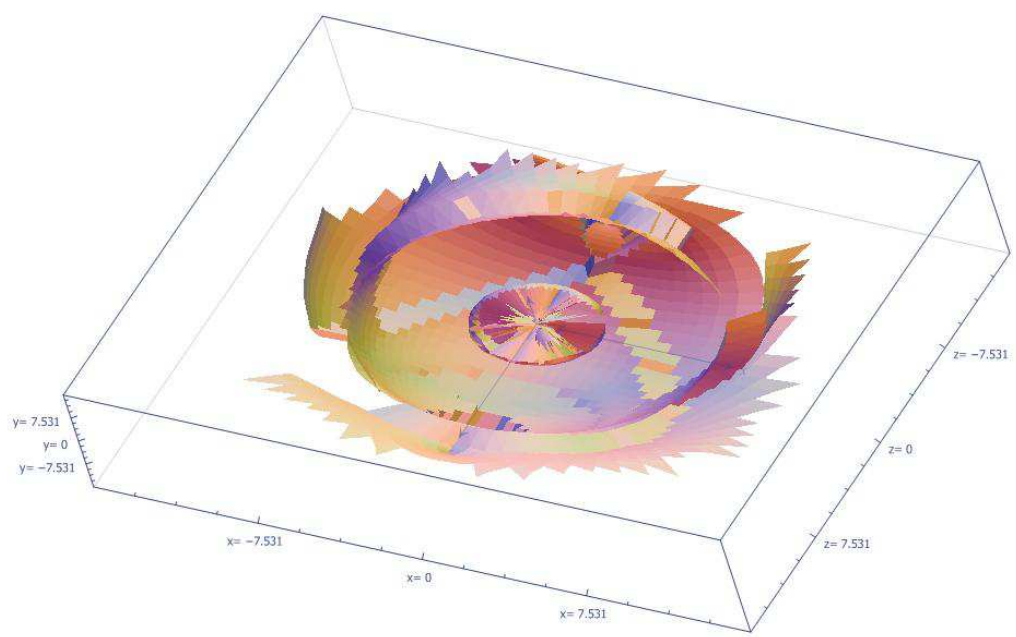

Figure 8. Nucleosome, nucleosome filament, annular domain. Chromosome tissue layers and chromosome mitosis begin.

\subsection{Partial Differentiation of High Dimensional Fiber Clusters and Soliton Wave Solutions of Protein 8 Polymer H1}

Binary first-order partial differential 8-mer $\mathrm{H} 1$ protein reflects the risk of mutation in chromosome transcription. Because there is an initial value in $\frac{d}{d \theta}\left(\operatorname{Sin}\left(\frac{3 \pi}{4}\right)+\operatorname{Sin}\left(\frac{3 \pi}{4}\right) \operatorname{Sin}\left(\frac{3 \pi}{4}\right)+2 \operatorname{Cos}^{3}\left(\theta+\frac{\pi}{4}\right)\right)$, the risk of cancer cells is reduced. Thus, we can see the absolute existence of cancer cells in the non-initial extended trigonometric series of second-order partial differential.

$$
\left\{\begin{aligned}
a=+\int & \operatorname{Cos}(z)\left[(-C) \operatorname{Sin}^{2}(b) \cdot \operatorname{Arcctg}\left(2 \operatorname{Sin}\left(y+\frac{\pi}{4}\right)+\frac{d}{d x}\left(\operatorname{Sin}(x)+\operatorname{Sin}^{2}(x)+2 \operatorname{Cos}^{3}\left(\frac{1}{2} x+\frac{\pi}{4}\right)\right)\right) d z+\int x \operatorname{Sin}(z) d z+\right. \\
& \left.+\operatorname{Sin}(z)\left((-C) \operatorname{Sin}^{2}(b) \cdot \operatorname{Arcctg}\left(2 \operatorname{Sin}\left(y+\frac{\pi}{4}\right)+\frac{d}{d x}\left(\operatorname{Sin}(x)+\operatorname{Sin}^{2}(x)+2 \operatorname{Cos}^{3}\left(\frac{1}{2} x+\frac{\pi}{4}\right)\right)\right)\right)+x \operatorname{Cos}(z)\right] \\
a=-\int & \operatorname{Cos}(z)\left[(-C) \operatorname{Sin}^{2}(b) \cdot \operatorname{Arcctg}\left(2 \operatorname{Sin}\left(y+\frac{\pi}{4}\right)+\frac{d}{d x}\left(\operatorname{Sin}(x)+\operatorname{Sin}^{2}(x)+2 \operatorname{Cos}^{3}\left(\frac{1}{2} x+\frac{\pi}{4}\right)\right)\right) d z+\int x \operatorname{Sin}(z) d z+\right. \\
& \left.+\operatorname{Sin}(z)\left((-C) \operatorname{Sin}^{2}(b) \cdot \operatorname{Arcctg}\left(2 \operatorname{Sin}\left(y+\frac{\pi}{4}\right)+\frac{d}{d x}\left(\operatorname{Sin}(x)+\operatorname{Sin}^{2}(x)+2 \operatorname{Cos}^{3}\left(\frac{1}{2} x+\frac{\pi}{4}\right)\right)\right)\right)+x \operatorname{Cos}(z)\right]
\end{aligned}\right.
$$

Formula 4. 2+1 dimension non-high-end hyperspherical

convex globular fibrous plexus chromosomal abnormalities of 
protein particles polypeptide spatial chain unwinding is similar to the soliton wave solution of the whole nonlinear partial differential equation system modified by the cell of chromosomal mitotic polypeptide spatial chain unwinding.

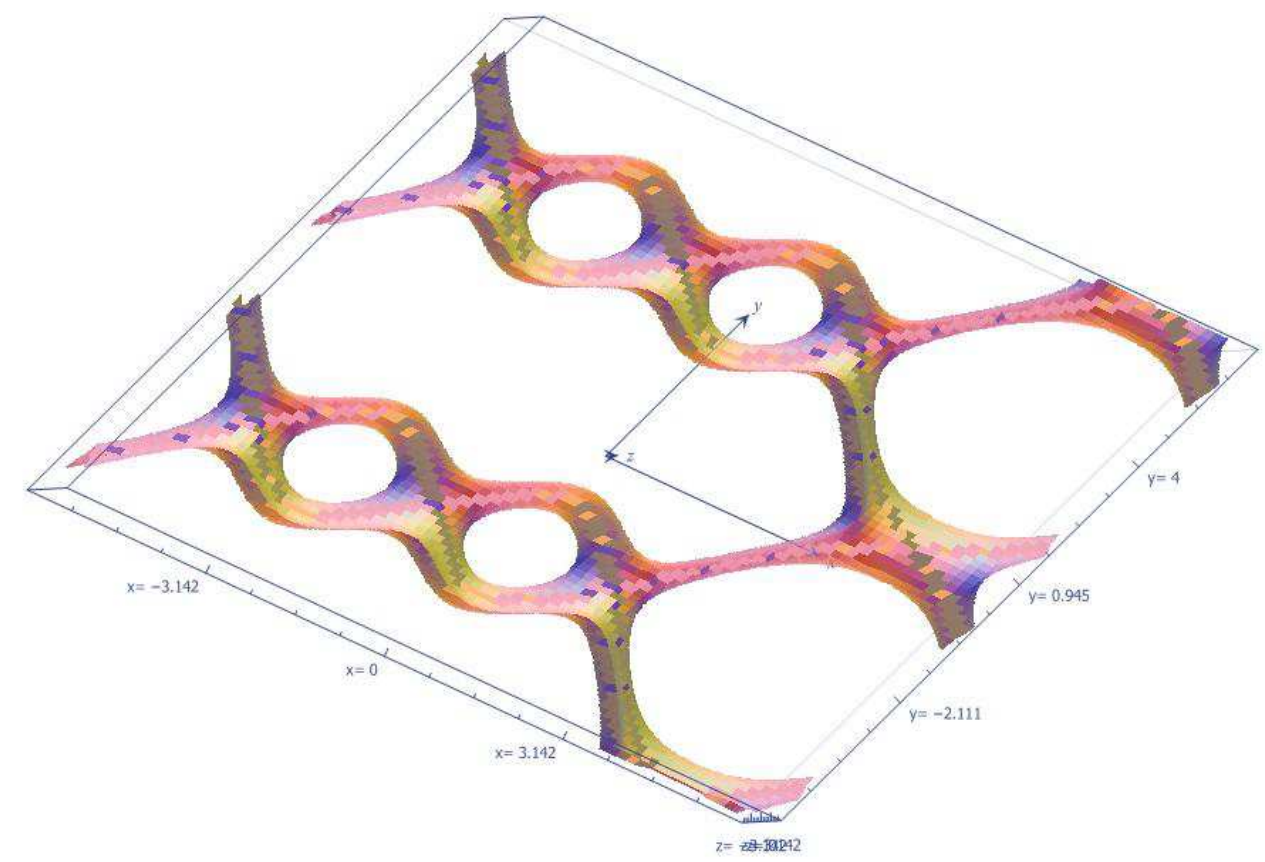

Figure 9. Solitary Wavelet Similarity Solution of Complex Normal Kernel Variables Constructed by Chromosome Mitosis and Polypeptide Spatial Unlinking.

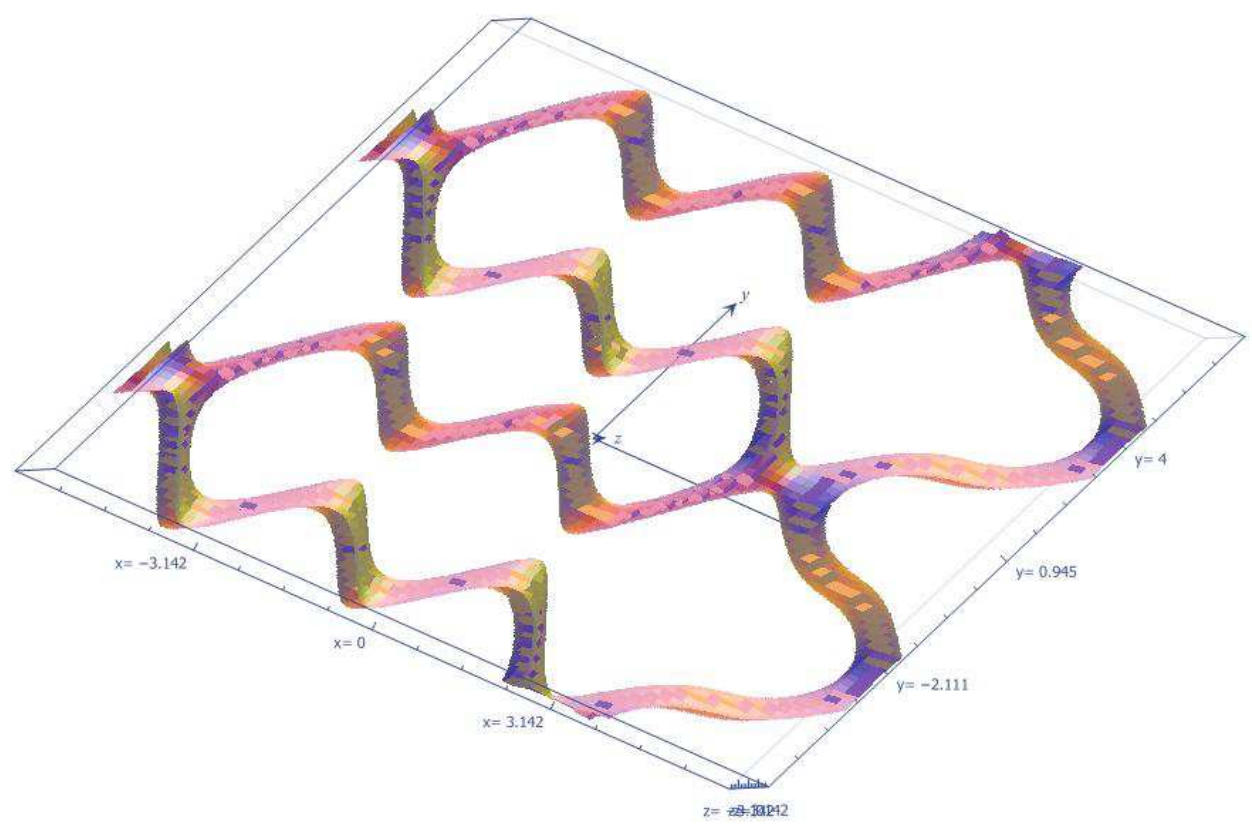

Figure 10. Solitary Wavelet Similarity Solution of Complex Abnormal Kernel Variables Constructed by Chromosome Mitosis and Polypeptide Spatial Unlinking.

\section{Genome Expression and Normal Cell Tissue Spatial Morphology of Protein Chain Combination and Chromosome Mitosis}

\subsection{Polypeptide Space Unchain and Mitotic Cell Modified Whole Solitary Wavelet of Chromosome}

4-dimensional super-high-end hyperspherical convex globular fibrous plexus, polypeptide spatial chain unwinding of normal chromosomal protein particles with initial boundary and global soliton wave range nuclear-controlled solution of normal cell tissue spatial morphology with weak microstructures modified by mitotic cells. 


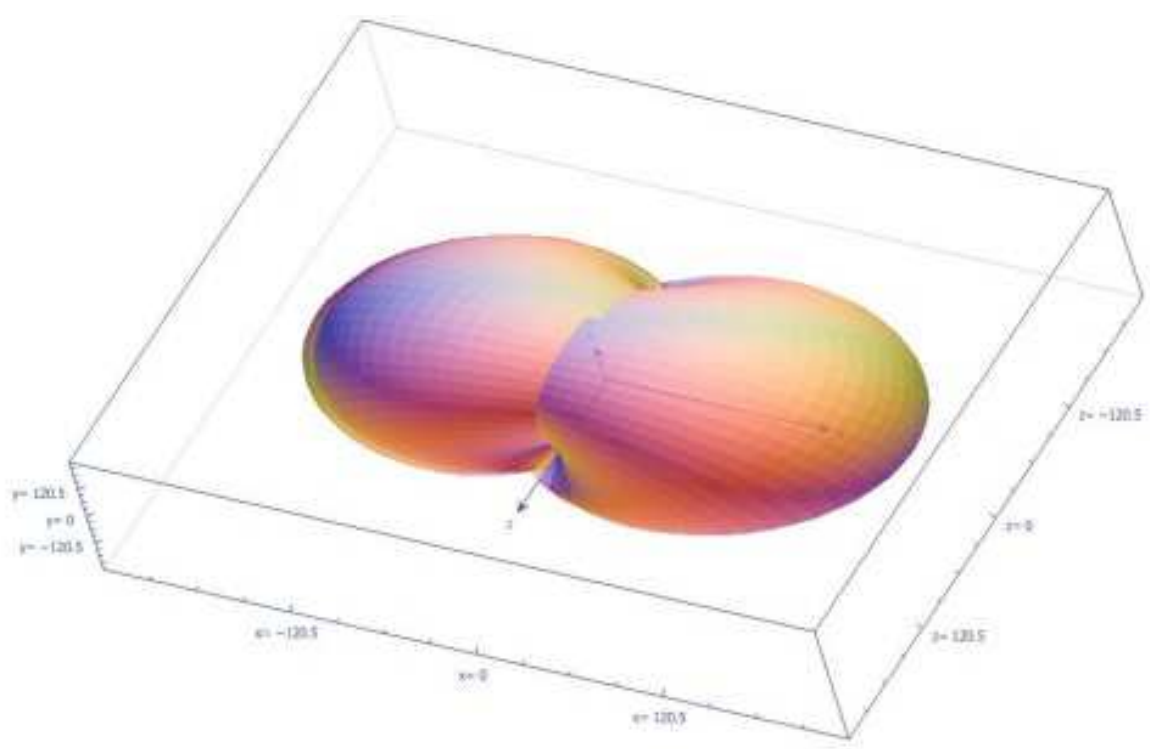

Figure 11. The cell modification of normal chromosome mitosis and the spatial morphology of normal cell tissue at the initial boundary.

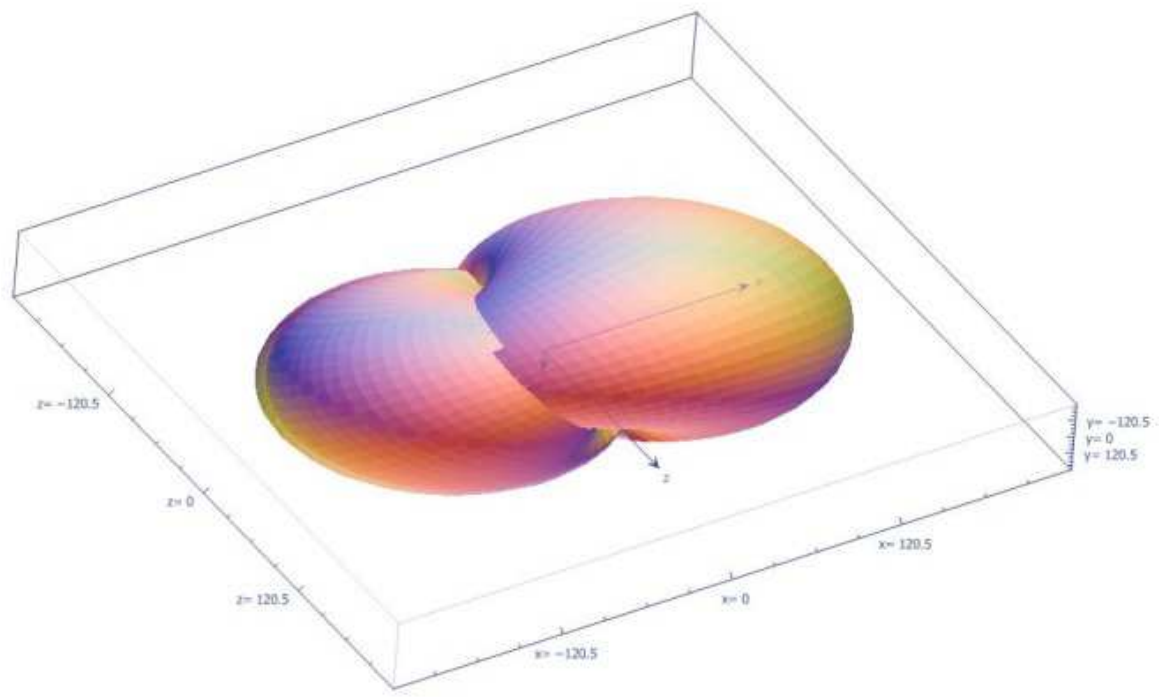

Figure 12. The cell modification of normal chromosome mitosis and the spatial morphology of normal cell tissue at the initial boundary.

$$
\left\{\begin{array}{l}
r_{(\theta, \varphi)}=\operatorname{ArcSin}\left[\frac{1}{-C \cdot \operatorname{Arcctg}\left[2 \operatorname{Sin}\left(\varphi+\frac{\pi}{4}\right)+\operatorname{Sin}\left(\frac{3 \pi}{4}\right)+\operatorname{Sin}^{2}\left(\frac{3 \pi}{4}\right)+\operatorname{Sin}^{3}\left(\frac{3 \pi}{4}\right)+2 \operatorname{Cos}^{3}\left(\theta+\frac{\pi}{4}\right)\right]}\right] \times \\
\operatorname{ArcCos}\left[\frac{1}{-C \cdot \operatorname{Arcctg}\left[2 \operatorname{Sin}\left(\varphi+\frac{\pi}{4}\right)+\operatorname{Sin}\left(\frac{3 \pi}{4}\right)+\operatorname{Sin}^{2}\left(\frac{3 \pi}{4}\right)+\operatorname{Sin}^{3}\left(\frac{3 \pi}{4}\right)+2 \operatorname{Cos}^{3}\left(\theta+\frac{\pi}{4}\right)\right]}\right]+2 \pi N_{1}+\frac{3 \pi}{4} \\
r_{(\theta, \varphi)}=\operatorname{ArcSin}\left[\frac{-C \cdot \operatorname{Arcctg}\left[2 \operatorname{Sin}\left(\varphi+\frac{\pi}{4}\right)+\operatorname{Sin}\left(\frac{3 \pi}{4}\right)+\operatorname{Sin}^{2}\left(\frac{3 \pi}{4}\right)+\operatorname{Sin}^{3}\left(\frac{3 \pi}{4}\right)+2 \operatorname{Cos}^{3}\left(\theta+\frac{\pi}{4}\right)\right]}{\operatorname{Arc} \operatorname{Cos}\left[\frac{1}{-C \cdot \operatorname{Arctg}\left[2 \operatorname{Sin}\left(\varphi+\frac{\pi}{4}\right)+\operatorname{Sin}\left(\frac{3 \pi}{4}\right)+\operatorname{Sin}^{2}\left(\frac{3 \pi}{4}\right)+\operatorname{Sin}^{3}\left(\frac{3 \pi}{4}\right)+2 \operatorname{Cos}^{3}\left(\theta+\frac{\pi}{4}\right)\right]}\right]+2 \pi N_{2}-\frac{5 \pi}{4}}\right. \\
{\left[\frac{1}{4}\right]}
\end{array}\right.
$$


Formula 5. Four-dimensional super-high-end hyperspherical convex globular fibrous plexus, polypeptide spatial chain unwinding of protein particles with initial boundary normal chromosomes and global soliton domain nuclear-controlled solution of normal cell tissue spatial morphology with weak microstructures modified by mitotic cells of chromosomes.

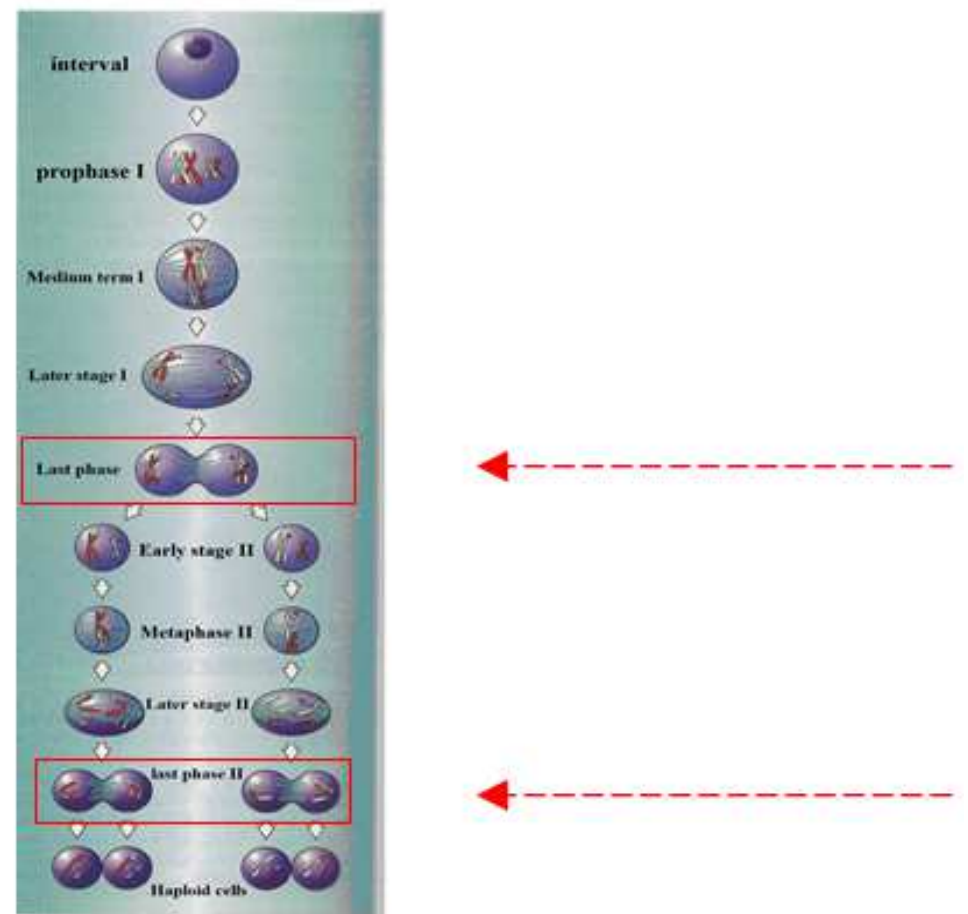

Figure 13. Normal chromosome mitosis cell modification initial boundary normal cell tissue spatial morphology electron microscopy.
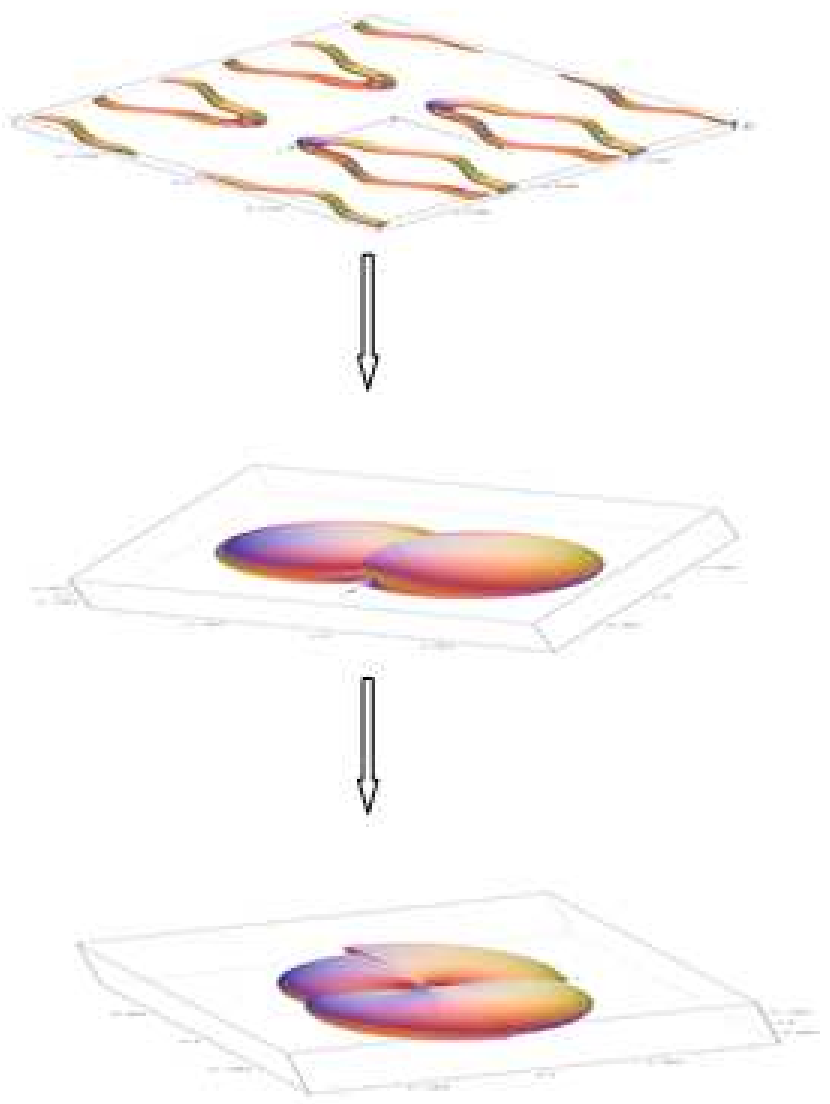

Figure 14. Normal chromosome mitosis cell modification initial boundary normal cell tissue spatial morphology 3D mathematical microscope. 


\subsection{Higher Dimensional Solitary Wavelets of Fibrous Plexus Play an Important Role in Replicating Cell Morphology}

4-dimensional super-high-end hyperglobular convex globular fibrous plexus normal chromosomal mitosis with initial boundary, forming the spatial morphology of haploid cells. Somatic cells of 4-dimensional super-high-end hyperglobular plexus were constructed by $3+1$-dimensional system of reduced-dimensional high-end hyperglobular plexus and polypeptide-normal chromosome protein-core. Comparing with the left figure above, we can see the importance of solitary wavelet in 4-dimensional super-high-end hyperspherical convex spherical fiber bundles.

\section{DNA Replication and Repair, Protein Particle Folding and Complex Polypeptide Space Unchain, as Well as the Space Structure of Helix Unraveling and Cell Modification, Cell Division}

\subsection{Analyzing the Three-dimensional Model of DNA Helix Unwrapping and the Core Homology of Polypeptide Space Chain Unwrapping}

DNA replication requires a three-dimensional model of DNA helix unwinding in cells, and the corresponding relationship between DNA core and polypeptide space chain unwinding.

The three-dimensional model of DNA molecule and helix resolving corresponds to the homology of polypeptide space chain resolving core.

$$
\left\{\begin{aligned}
a= & +\int \operatorname{Sin}\left(z+\frac{\pi}{4}\right)\left[(-C) \operatorname{Sin}^{2}(b) \cdot \operatorname{Arcctg}\left[\left(2 \operatorname{Cos}^{3}\left(\frac{1}{2} x+\frac{\pi}{4}\right)+2 \operatorname{Sin}\left(y+\frac{\pi}{4}\right)+\operatorname{Sin}(x)+\operatorname{Sin}^{2}(x)\right)\right] d z+\left(-\operatorname{Cos}\left(z+\frac{\pi}{4}\right)\right) .\right. \\
& \left.\cdot\left((-C) \operatorname{Sin}^{2}(b) \cdot \operatorname{Arcctg}\left[\left(2 \operatorname{Cos}^{3}\left(\frac{1}{2} x+\frac{\pi}{4}\right)+2 \operatorname{Sin}\left(y+\frac{\pi}{4}\right)+\operatorname{Sin}\left(\frac{3 \pi}{4}\right)+\operatorname{Sin}(x)+\operatorname{Sin}^{2}(x)\right)\right]\right)\right]+\left(\int x \operatorname{Sin}(z) d z+x \operatorname{Cos}(z)\right) \\
a= & -\int \operatorname{Sin}\left(z+\frac{\pi}{4}\right)\left[(-C) \operatorname{Sin}^{2}(b) \cdot \operatorname{Arcctg}\left[\left(2 \operatorname{Cos}^{3}\left(\frac{1}{2} x+\frac{\pi}{4}\right)+2 \operatorname{Sin}\left(y+\frac{\pi}{4}\right)+\operatorname{Sin}(x)+\operatorname{Sin}^{2}(x)\right)\right] d z-\left(-\operatorname{Cos}\left(z+\frac{\pi}{4}\right)\right) .\right. \\
& \left.\cdot\left((-C) \operatorname{Sin}^{2}(b) \cdot \operatorname{Arcctg}\left[\left(2 \operatorname{Cos}^{3}\left(\frac{1}{2} x+\frac{\pi}{4}\right)+2 \operatorname{Sin}\left(y+\frac{\pi}{4}\right)+\operatorname{Sin}\left(\frac{3 \pi}{4}\right)+\operatorname{Sin}(x)+\operatorname{Sin}^{2}(x)\right)\right]\right)\right]-\left(\int x \operatorname{Sin}(z) d z+x \operatorname{Cos}(z)\right)
\end{aligned}\right.
$$

Formula 6. 3+1-dimensional system dimension-reduced high-end hyperspherical convex spherical fibrous cluster protein particle Space folding and protein particle space construction, and cell modification is the protein particle space construction, folding-related. with complex (core variables) polypeptide space Chain unwinding structure solitary wavelet value domain nuclear-controlled similar solution.

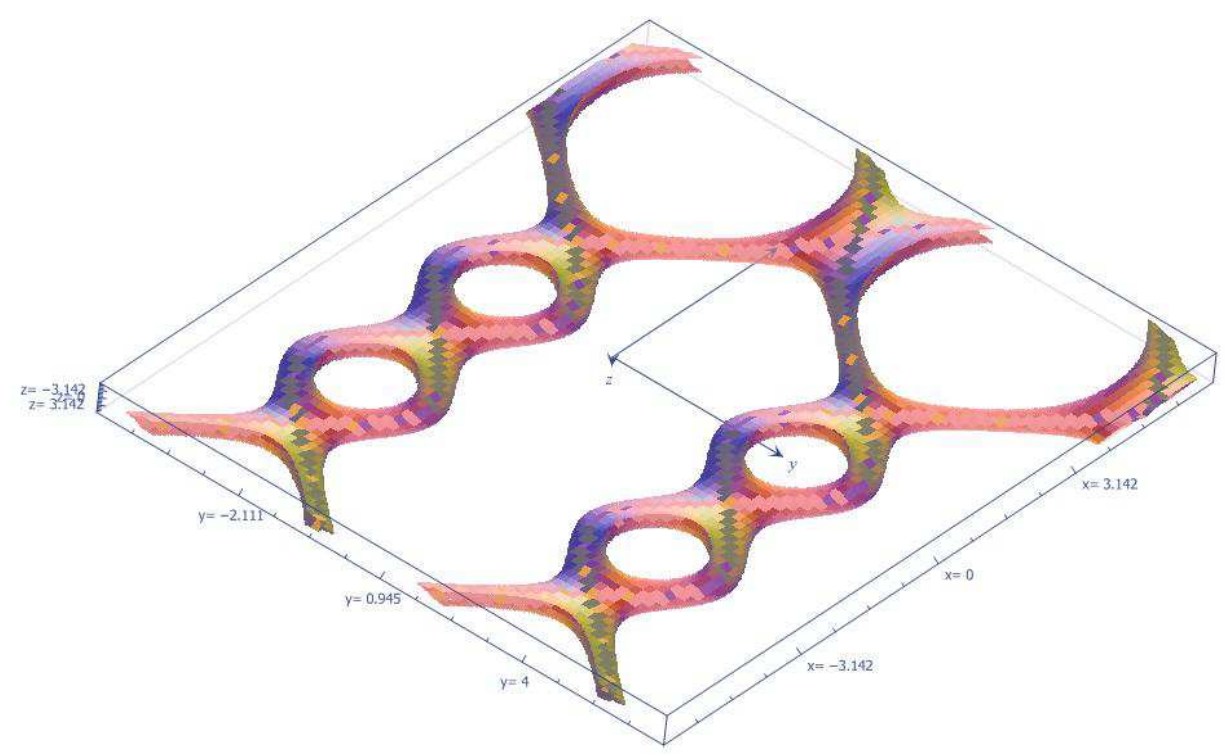

Figure 15. The three-dimensional model of DNA molecule and helix unwinding corresponds to the homology of polypeptide space chain unwinding core. 


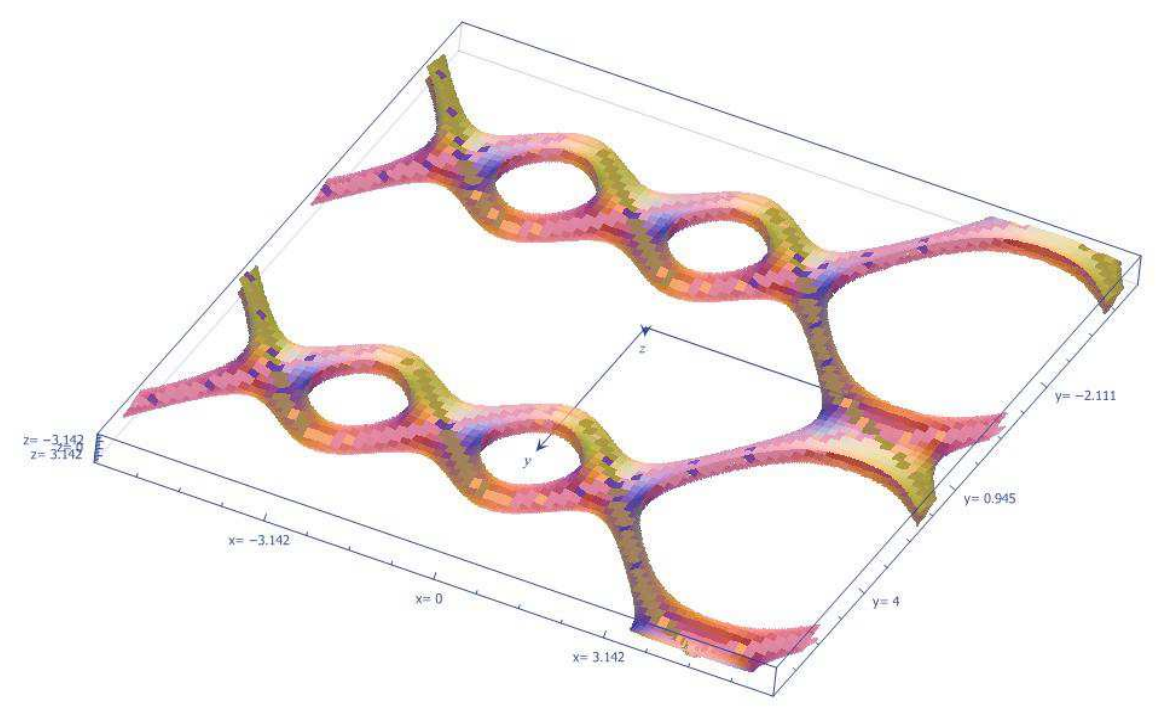

Figure 16. The three-dimensional model of DNA molecule and helix unwinding corresponds to the homology of polypeptide space chain unwinding core.

\subsection{The Homology of the Nuclei in the Spatial}

Chain-unlocking of Polypeptides Directly Affects the

Core Morphology of the Spatial Chain-unlocking of

Polypeptides

The semi-reserved replication form of DNA helix unwrapping three-dimensional model was used to compare the Three-dimensional images of polypeptide space unlinking core homology mentioned above. It is found that DNA helix unwinding has a direct impact on polypeptide space chain unwinding core morphology.

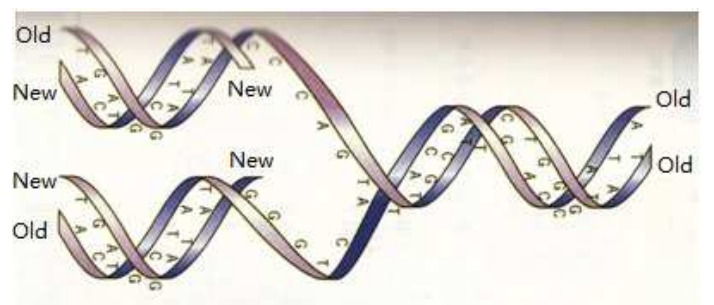

Figure 17. DNA helix unwinding affects the core morphology of polypeptide space chain unwinding.

DNA molecule, together with various purified proteins, forms the above-mentioned polypeptide space unchain core morphology and the binding sites of transcriptional regulatory DNA molecule binding proteins, which provide direction for the beginning of replication.

\section{DNA Unravels Double Helix and Separates Double Strands, and Replicates Bi-directionally and Semi-Reserved with Circular Chromosomes}

\subsection{The Complex Kernel Variables of Solitary Wavelets of High-end Fibre Bundles in 3+1 Dimension Are Related to Deep Chain-breaking}

$3+1$ dimension-reduced high-end hyperspherical convex fiber bundles, protein particle Space folding and protein particle space construction, and cell modification, is related to protein particle space construction and folding. The polypeptide with complex (core variables) space depth unravels the chain structure, forming a similar solution of the soliton wave range nuclear control of single-chain protein particles.

Ring chromosomes are duplicated by two-way semi-reserved replication. Two duplication double helixes are separated from each other, and two duplication forks move from the same starting point to the opposite direction. When two replication forks meet at the relative points on the ring, the replication terminates.

$$
\left\{\begin{aligned}
a= & +\int \operatorname{Sin}\left(z+\frac{\pi}{4}\right)\left[(-C) \operatorname{Sin}^{2}(b) \cdot \operatorname{Arcctg}\left[\left(2 \operatorname{Cos}^{3}\left(\frac{1}{2} x+\frac{\pi}{4}\right)+2 \operatorname{Sin}\left(y+\frac{\pi}{4}\right)+\operatorname{Sin}(x)+\operatorname{Sin}^{2}(x)+\operatorname{Sin}^{3}(x)\right)\right] d z+\left(-\operatorname{Cos}\left(z+\frac{\pi}{4}\right)\right)\right. \\
& \left.\cdot\left((-C) \operatorname{Sin}^{2}(b) \cdot \operatorname{Arctg}\left[\left(2 \operatorname{Cos}^{3}\left(\frac{1}{2} x+\frac{\pi}{4}\right)+2 \operatorname{Sin}\left(y+\frac{\pi}{4}\right)+\operatorname{Sin}\left(\frac{3 \pi}{4}\right)+\operatorname{Sin}(x)+\operatorname{Sin}^{2}(x)+\operatorname{Sin}^{3}(x)\right)\right]\right)\right]+\left(\int x \operatorname{Sin}(z) d z+x \operatorname{Cos}(z)\right) \\
a= & -\int \operatorname{Sin}\left(z+\frac{\pi}{4}\right)\left[(-C) \operatorname{Sin}^{2}(b) \cdot \operatorname{Arcctg}\left[\left(2 \operatorname{Cos}^{3}\left(\frac{1}{2} x+\frac{\pi}{4}\right)+2 \operatorname{Sin}\left(y+\frac{\pi}{4}\right)+\operatorname{Sin}(x)+\operatorname{Sin}^{2}(x)+\operatorname{Sin}^{3}(x)\right)\right] d z+\left(-\operatorname{Cos}\left(z+\frac{\pi}{4}\right)\right)\right. \\
& \left.\cdot\left((-C) \operatorname{Sin}^{2}(b) \cdot \operatorname{Arcctg}\left[\left(2 \operatorname{Cos}^{3}\left(\frac{1}{2} x+\frac{\pi}{4}\right)+2 \operatorname{Sin}\left(y+\frac{\pi}{4}\right)+\operatorname{Sin}\left(\frac{3 \pi}{4}\right)+\operatorname{Sin}(x)+\operatorname{Sin}^{2}(x)+\operatorname{Sin}^{3}(x)\right)\right]\right)\right]-\left(\int x \operatorname{Sin}(z) d z+x \operatorname{Cos}(z)\right)
\end{aligned}\right.
$$



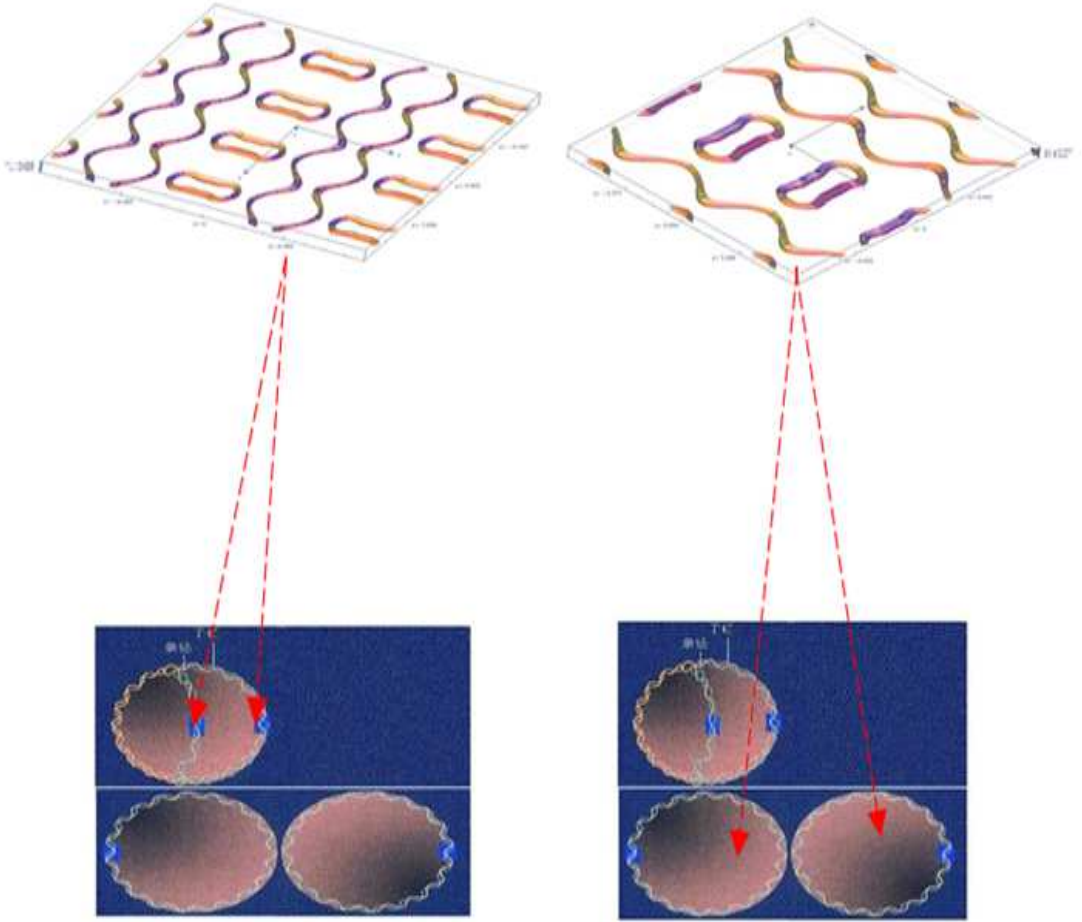

Figure 18. Spatial folding of protein particles is similar to the solitary wave solution of the structure of the nuclear variable polypeptide spatial chain. Ring chromosomes were duplicated by two-way Semi-Reserved replication, and the two duplicated double helixes were separated from each other.

Formula 7. 3+1 dimension-reduced high-end hyperspherical convex spherical fiber bundles, protein particle Space folding and protein particle space construction, and cell modification, is the protein particle space construction, folding-related, with complex (core variables) polypeptide space depth unraveling chain structure, and form a single-chain shape of protein particle space transformation of the whole nonlinear partial differential equation group-solitary wavelet Similar solutions.

\subsection{Duplication Fork and Bidirectional Duplication, Cyclic DNA Double Helix with Many Proteins Binding to the Cyclic Chromosome}

The starting point of chromosomal replication of cyclic DNA double helix binding to many proteins is a sequence called oric, with which many proteins bind and initiate the replication process. This regulatory sequence serves as a binding site for sequence-specific DNA-binding proteins.

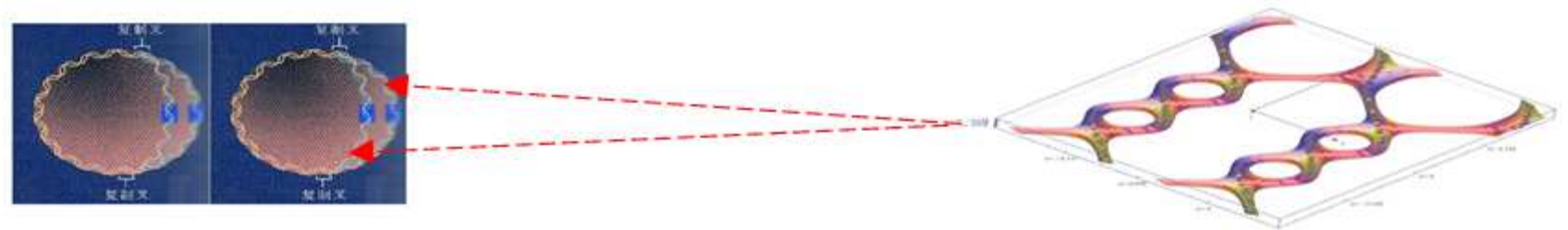

Figure 19. Duplication fork and bidirectional duplication, cyclic DNA double helix with many proteins binding to the cyclic chromosome.

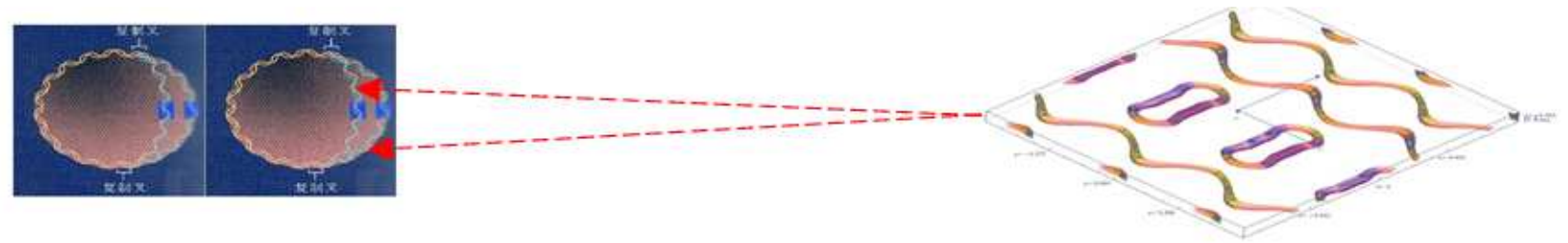

Figure 20. Duplication fork and bidirectional duplication, cyclic DNA double helix with many proteins binding to the cyclic chromosome. Cyclic chromosome that binds to a protein by a double helix of cyclic DNA.

Ring chromosomes begin at the bifurcation point, double helix DNA is divided into daughter and affinity chains. Replication ends when the replication fork begins to meet at the relative point on the ring, and two cells are formed. There are a large number of proteins on the circular chromosome or double helix DNA, and forming the necessary polypeptide for cells. 

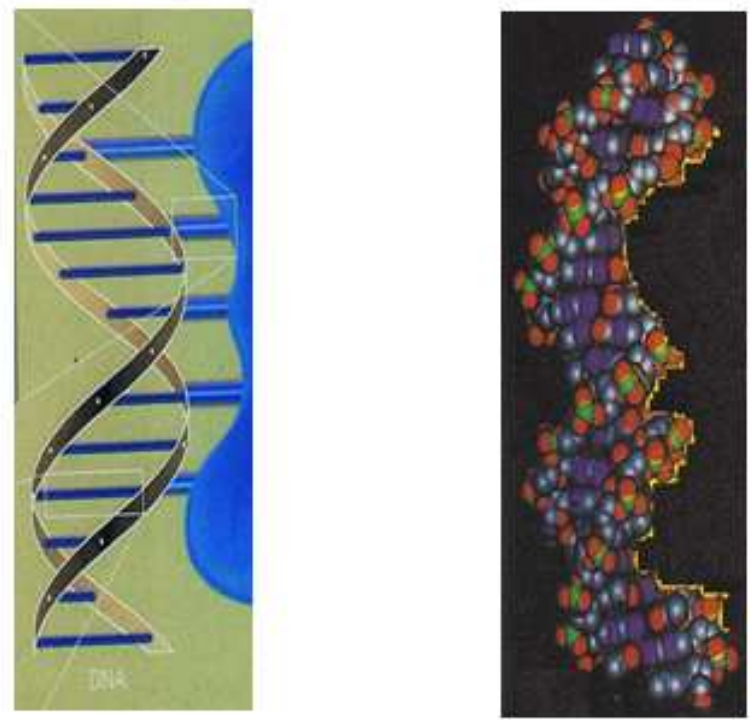

Figure 21. The double helix configuration of DNA and protein molecules (particles).

\subsection{Non-covalent ion Bonds Play an Important Role in the Binding of DNA to Protein Molecules (Particles) and Protein Molecules (Particles) to DNA Helix Unwinding and Polypeptide Space Chain Unwinding}

Protein molecules bind to DNA molecules and form ionic bonds between positively charged nitrogen atoms in proteins and negatively charged oxygen atoms in DNA. (1) DNA binds to protein molecules (particles) to form a DNA-like double helix structure. Kernel homology of unchains in polypeptide space, and DNA helix unwinding directly affects the nuclear morphology of the space chain unwinding of polypeptides.
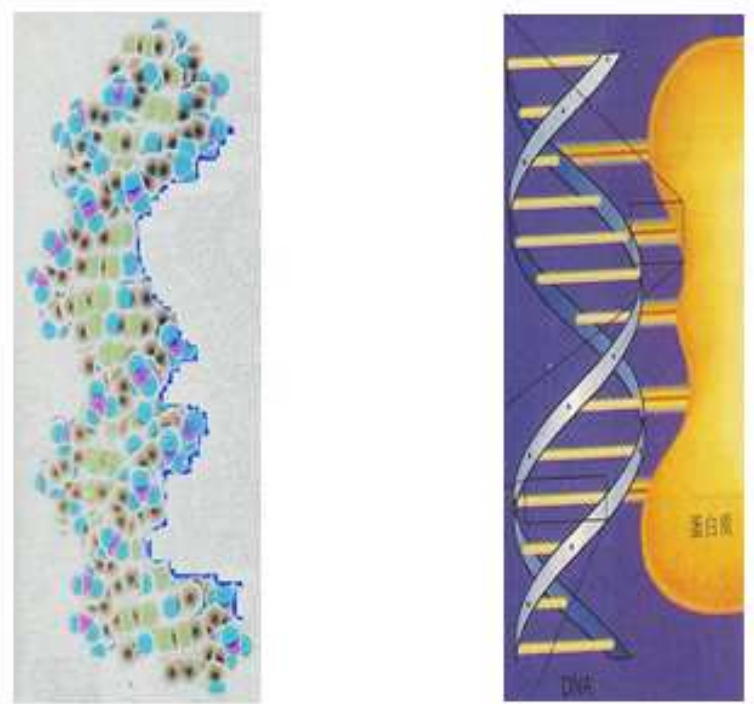

Figure 22. The double helix configuration of DNA and protein molecules (particles).

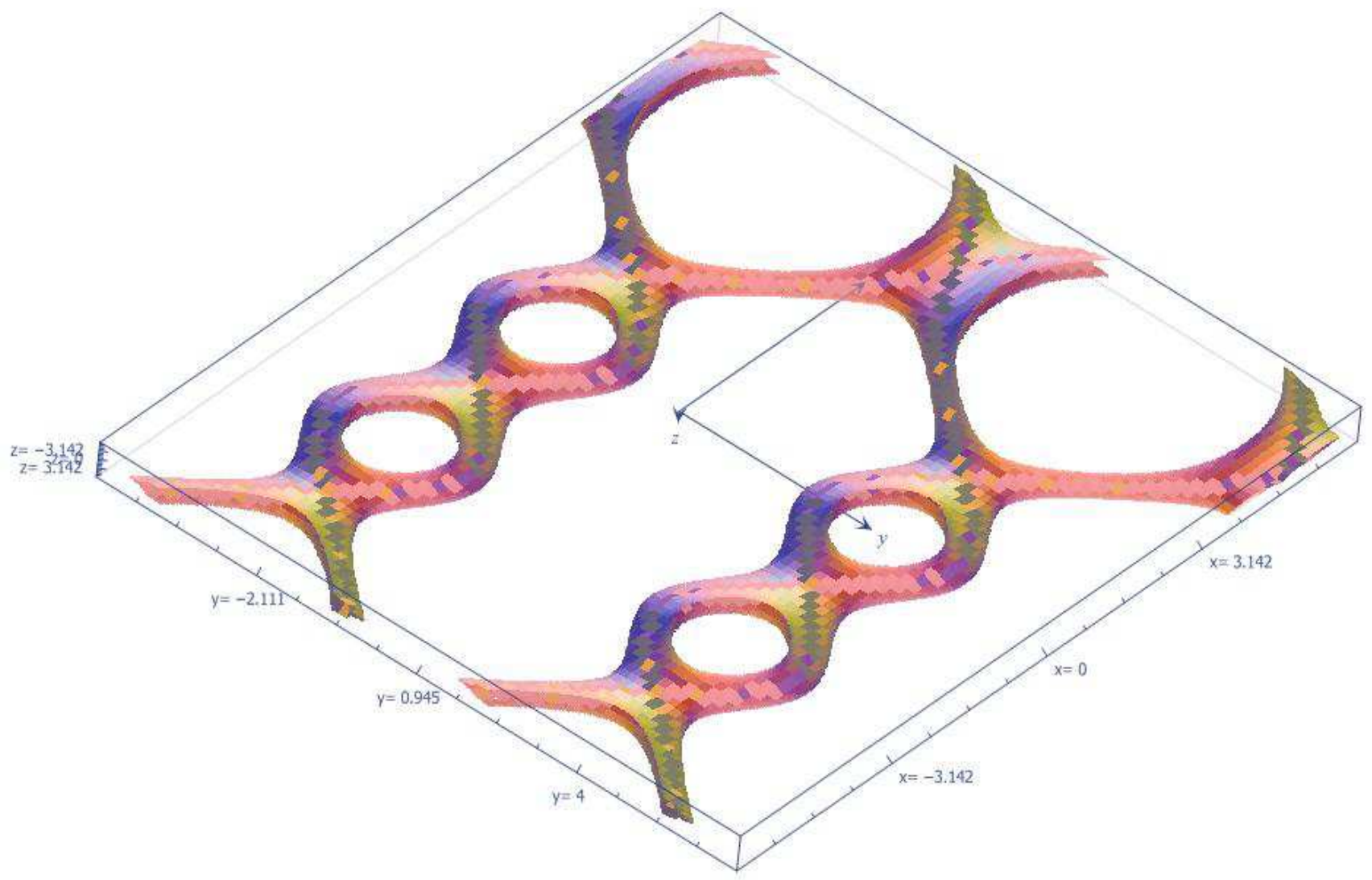

Figure 23. Core morphology of DNA helix-unwrapping polypeptide space chain-unwrapping. 


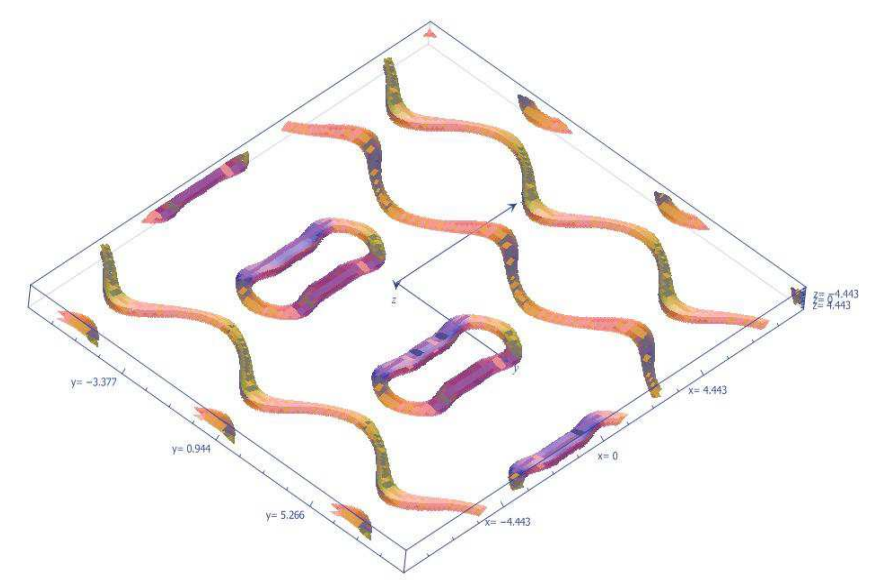

Figure 24. Core morphology of DNA helix-unwrapping polypeptide space chain-unwrapping.

\section{Complex Polypeptide Composed of Plasma Membrane of Muscle Cell and Myofibrillar Protein Chain}

Macroscopic non-holomorphic secondary micro-incremental equilibrium spatial structure of the combination of muscle cell plasma membrane and myofibrin chain, as well as the complex structure of twisted and folded polypeptide chains of the combination of muscle cell plasma membrane, protein sarcoplasmic reticulum membrane cannula and myofibrin chain.

The general solution of holistic protein particles in the macroscopic non-holomorphic secondary micro-incremental equilibrium spatial structure of the combination of muscle cell plasma membrane and myofibrillar protein chain.

$$
\left\{\begin{aligned}
a=+ & \int \operatorname{Cos}(z)\left[-C \cdot \operatorname{Sin}^{2}(b)\left(\operatorname{Sec}^{2}\left(y+\frac{\pi}{4}\right) \operatorname{Cos}^{2}\left(\frac{x}{2}+\frac{\pi}{4}\right)+2 \operatorname{Cos}^{3}\left(\frac{x}{2}+\frac{\pi}{4}\right)\right) d z+\right. \\
& \left.\int x \operatorname{Sin}(z) d z-C \cdot \operatorname{Sin}(z) \operatorname{Sin}^{2}(b)\left(\operatorname{Sec}^{2}\left(y+\frac{\pi}{4}\right) \operatorname{Cos}^{2}\left(\frac{x}{2}+\frac{\pi}{4}\right)+2 \operatorname{Cos}^{3}\left(\frac{x}{2}+\frac{\pi}{4}\right)\right)+x \operatorname{Cos}(z)\right] \\
a= & -\int \operatorname{Cos}(z)\left[-C \cdot \operatorname{Sin}^{2}(b)\left(\operatorname{Sec}^{2}\left(y+\frac{\pi}{4}\right) \operatorname{Cos}^{2}\left(\frac{x}{2}+\frac{\pi}{4}\right)+2 \operatorname{Cos}^{3}\left(\frac{x}{2}+\frac{\pi}{4}\right)\right) d z+\right. \\
& \left.\int x \operatorname{Sin}(z) d z-C \cdot \operatorname{Sin}(z) \operatorname{Sin}^{2}(b)\left(\operatorname{Sec}^{2}\left(y+\frac{\pi}{4}\right) \operatorname{Cos}^{2}\left(\frac{x}{2}+\frac{\pi}{4}\right)+2 \operatorname{Cos}^{3}\left(\frac{x}{2}+\frac{\pi}{4}\right)\right)+x \operatorname{Cos}(z)\right]
\end{aligned}\right.
$$

Formula 8. 2+1 dimension high-end hyperglobular convex globular fibrous plexus has a similar solution to the holistic protein particles of the macroscopic non-holomorphic secondary micro-incremental equilibrium spatial structure of the plasma membrane and myofibrillar protein chains of muscle cells.

\subsection{The Terminal Globule Is Very Close to the Plasma Membrane of Muscle Cells, and Neurotransmitters Released from Synaptic Vesicles of Presynaptic Neurons Bind to Receptors on the Surface of Muscle Cells}

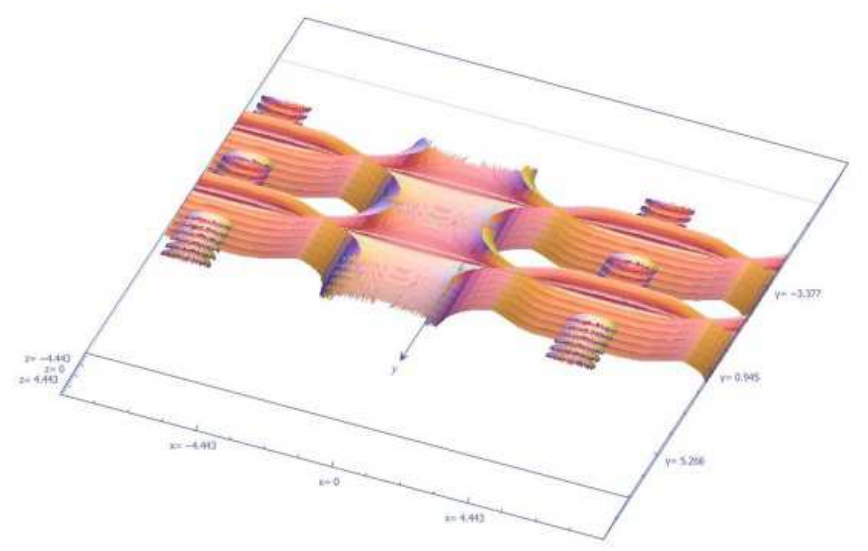

Figure 25. The Solitary Wavelet Similarity Solution of the Combination of the Membrane and Fibrin Chain of Muscle Cells. 


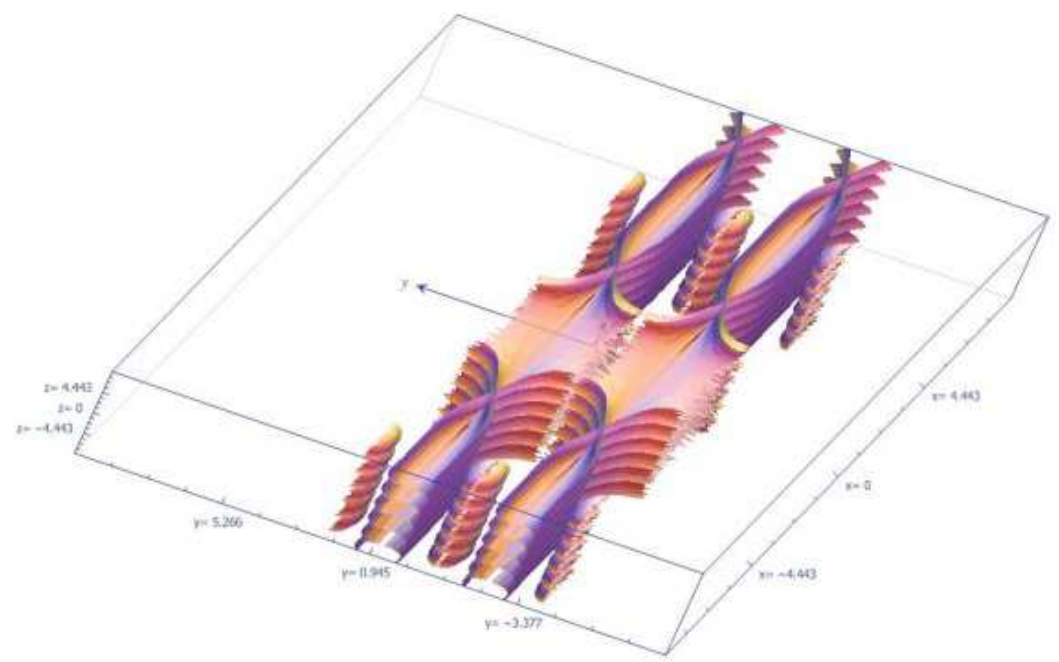

Figure 26. The Solitary Wavelet Similarity Solution of the Combination of the Membrane and Fibrin Chain of Muscle Cells.

\subsection{The Terminal Bulb of Neurons Attaches Slightly to the Muscle Fibers}

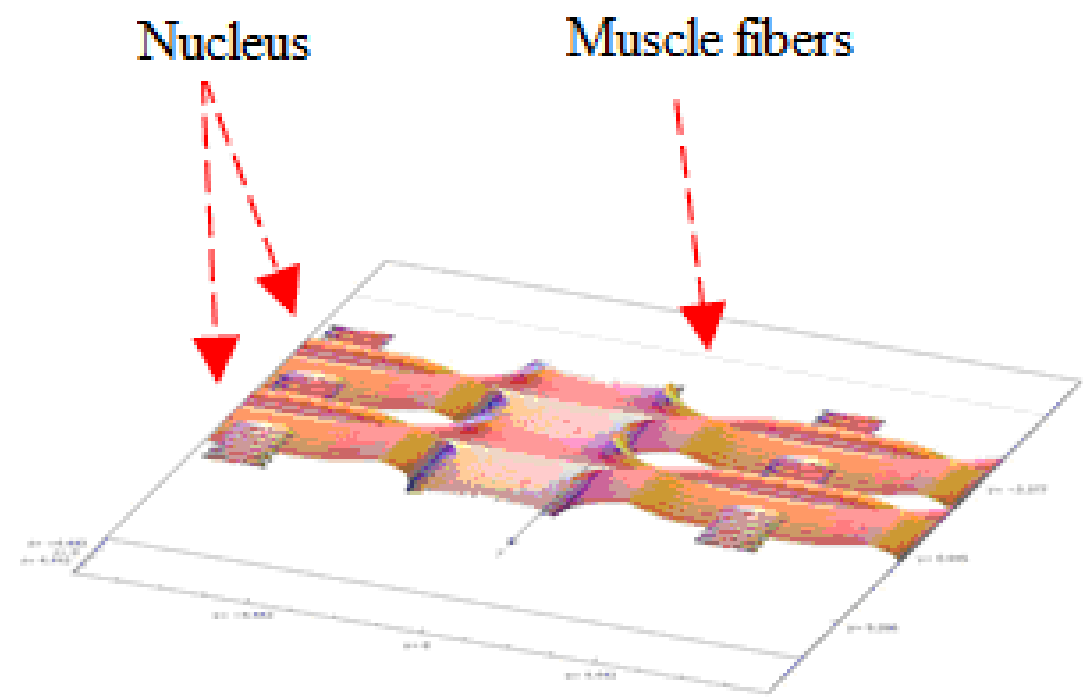

Figure 27. The Solitary Wavelet Similarity Solution of the Combination of the Membrane and Fibrin Chain of Muscle Cells.

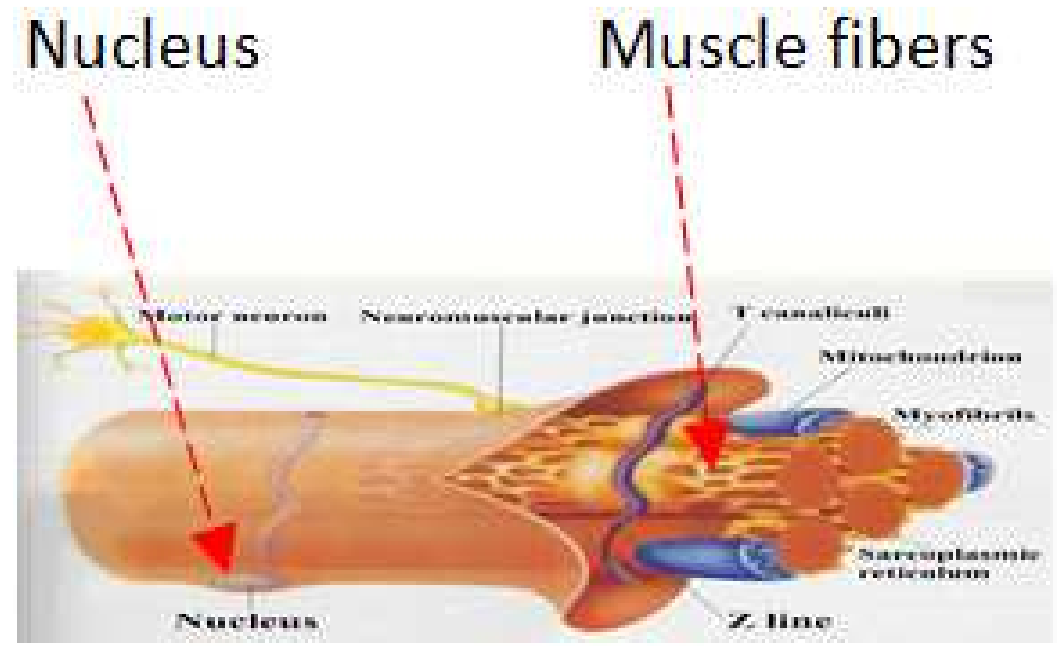

Figure 28. The Solitary Wavelet Similarity Solution of the Combination of the Membrane and Fibrin Chain of Muscle Cells. 


\section{The Polypeptide Chains of Fibrin, Globulin and Myoglobin Twist and Fold into Complex Shapes}

\subsection{Fibrin and Myoglobin Are Folded and Packed in a Ring}

Most proteins are classified according to their overall conformation: highly stretched fibrins (most of the proteins of extracellular structural substances are fibrins) and globulins (most of the proteins in cells are globulins). Polypeptide chains of myoglobin (globulin) twist and fold into complex shapes, in which polypeptides are folded and packaged. This molecule can't find the same regularity and symmetry as the DNA double helix structure. The coiled spiral structure exists in fibrin, such as myosin in muscle.
It consists of several alpha helical chains intertwined with each other, and there is a protein base order $\alpha / \beta$ barrel. The 8 plates of the barrel are composed of $8 \beta$ chains. These 8 parallel $\beta$ chains are located inside the barrel and form the core of the protein. The $8 \beta$ chains are connected by an $\alpha$ helical structure on the surface of the barrel.

\subsection{Global Solitary Wavelet Similarity Solutions for the Protein Chain Combination Myosin Alpha/Beta Barrel of Convex Globular Fiber Plexus}

4-dimensional super-high-end hyperspherical convex spherical fibrous plexus, the general solution of the solitary wave-like solution of the whole protein particle (polypeptide) in the $\alpha / \beta$ barrel (first-order differential) of myosin combined with the protein chain.

$$
\left\{\begin{array}{l}
r_{(\theta, \varphi)}=\operatorname{ArcSin}\left[\frac{1}{-C \cdot \operatorname{Sin}^{2}(b)\left[\operatorname{Sec}^{2}\left(\varphi+\frac{\pi}{4}\right) \frac{d}{d \theta}\left(\operatorname{Cos}^{2}\left(\frac{\theta}{2}+\frac{\pi}{4}\right)\right)+\frac{d}{d \theta}\left(2 \operatorname{Cos}^{3}\left(\frac{\theta}{2}+\frac{\pi}{4}\right)\right)\right]}\right] \operatorname{Arc} \operatorname{Cos}\left[\frac{1}{-C \cdot \operatorname{Sin}^{2}(b)\left[\operatorname{Sec}^{2}\left(\varphi+\frac{\pi}{4}\right) \frac{d}{d \theta}\left(\operatorname{Cos}^{2}\left(\frac{\theta}{2}+\frac{\pi}{4}\right)++\frac{d}{d \theta}\left(2 \operatorname{Cos}^{3}\left(\frac{\theta}{2}+\frac{\pi}{4}\right)\right)\right]\right.}\right]+2 \pi N_{1}+\frac{3 \pi}{4} \\
r_{(\theta, \varphi)}=\operatorname{ArcSin}\left[\frac{1}{-C \cdot \operatorname{Sin}^{2}(b)\left[\operatorname{Sec}^{2}\left(\varphi+\frac{\pi}{4}\right) \frac{d}{d \theta}\left(\operatorname{Cos}^{2}\left(\frac{\theta}{2}+\frac{\pi}{4}\right)\right)+\frac{d}{d \theta}\left(2 \operatorname{Cos}^{3}\left(\frac{\theta}{2}+\frac{\pi}{4}\right)\right)\right]}\right] \operatorname{Arc} \operatorname{Cos}\left[\frac{1}{-C \cdot \operatorname{Sin}^{2}(b)\left[\operatorname{Sec}^{2}\left(\varphi+\frac{\pi}{4}\right) \frac{d}{d \theta}\left(\operatorname{Cos}^{2}\left(\frac{\theta}{2}+\frac{\pi}{4}\right)\right)+\frac{d}{d \theta}\left(2 \operatorname{Cos}^{3}\left(\frac{\theta}{2}+\frac{\pi}{4}\right)\right)\right]}\right]+2 \pi N_{2}-\frac{5 \pi}{4}
\end{array}\right.
$$

Formula 9. 4-dimensional super-high-end hyperspherical convex spherical fibrous plexus, the general solution of the solitary wave-like solution of the whole protein particle (polypeptide) in the $\alpha / \beta$ barrel (first-order differential) of myosin combined with the protein chain.

\section{The soliton of the $\alpha / 8$ barrel of 8 plates of the barrel.}

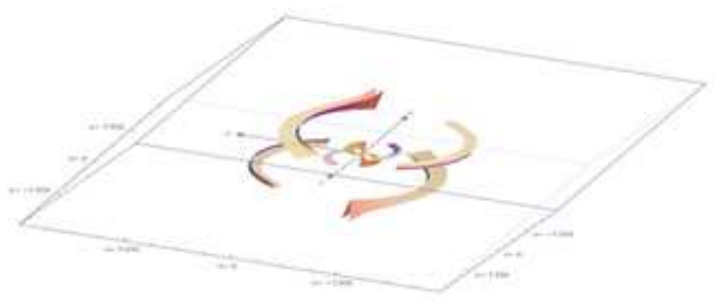

Figure 29. Myosin-protein motif of protein chain combination.

\section{The soliton of the $\alpha / \beta$ barrel of 8 plates of the barrel.}

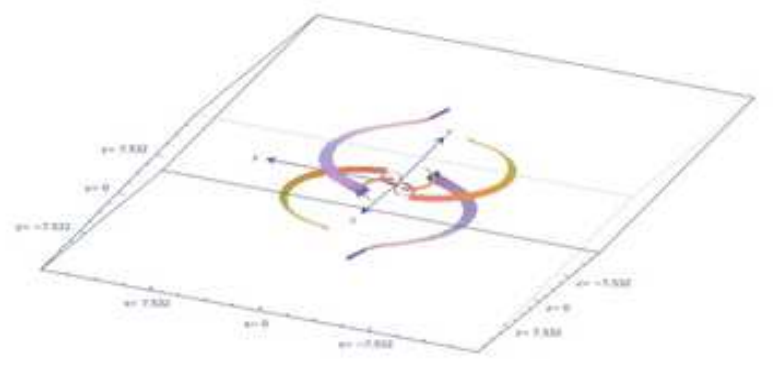

Figure 30. Myosin-protein motif of protein chain combination. 
4-dimensional super-high-end hyperspherical convex globular fibrous plexus, the alpha/beta barrel of myosin composed of protein chains (the first-order differential does not constitute a tumor-type structure) solitary wavelet, especially in muscle fibers and myosin. Second-order differential is a high-dimensional fibrous plexus with high cancer risk.

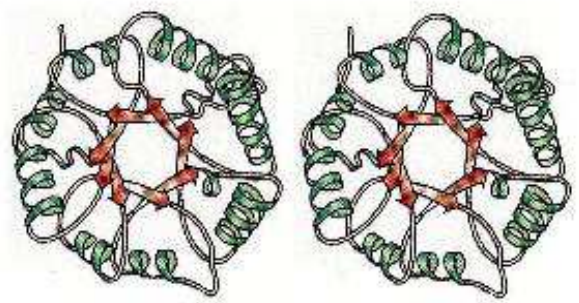

Figure 31. Myosin-protein motif of protein chain combination. 8 plates of the barrel, $\alpha / \beta$ barrels.

\section{Conclusion}

In solving the abstract model of molecular cell biology, primitive innovative mathematics can better and deeply intervene in gene mapping and gene sequencing.

From computer information science to life science, we deduce the normal cell space morphology of normal chromosome mitotic modification and initial boundary at the molecular level. DNA unravels double helix and separates double strands, and makes bi-directional Semi-Reserved replication with ring chromosomes; the two duplicate double helixes separate from each other, duplicate fork and duplicate bi-directionally. Cyclic DNA double helix is a cyclic chromosome that binds to many proteins. From computer information science and intelligent Mathematics - high-end hyperspherical convex spherical fiber bundles, the similarity solution of high-dimensional solitary wavelet with deep Chain unwinding structure of polypeptide space is formed, which embodies its inherent special non-linear wave form.

\section{References}

[1] C. Rogers W. K. Schief, Bäcklund and Darboux Transformations: Geometry and Modern Applications in Solition Theory, first published by Cambridge University, 2015 $1-292$.

[2] Chen Zhonghu, Lie group guidance, Higher Education Press, 1997: 1-334.

[3] Ding Peizhu Wang Yi, Group and its Express, Higher Education Press, 1999: 1-468.

[4] E. M. Chirka, Complex Analytic Sets Mathematics and Its Applications, Kluwer Academic Publishers Gerald Karp, Cell and Molecular Biology: Concepts and Experiments (3e), Higher Education Press, 2005: 1-792.

[5] Gong Sheng, Harmonic Analysis on Typical Groups Monographs on pure mathematics and Applied Mathematics Number twelfth, Beijing China, Science Press, 1983: 1-314.
[6] $\mathrm{Gu}$ chaohao $\mathrm{Hu}$ Hesheng Zhou Zixiang, DarBoux Transformation in Solition Theory and Its Geometric Applications (The second edition), Shanghai science and technology Press, 1999, 2005: 1-271.

[7] Jari Kaipio Erkki Somersalo, Statistical and Computational Inverse Problems With 102 Figures, Spinger.

[8] Lou Senyue Tang Xiaoyan, Nonlinear Mathematical Physics Method, Beijing China, Science Press, 2006: 1-365.

[9] Numerical Treatment of Multi-Scale Problems Porceedings of the $13^{\text {th }}$ GAMM-Seminar, Kiel, January 24-26, 1997 Notes on Numerical Fluid Mechanics Volume 70 Edited By WolfGang HackBusch and Gabriel Wittum.

[10] Qiu Chengtong Sun Licha, Differential Geometry Monographs on pure mathematics and Applied Mathematics Number eighteenth, Beijing China, Science Press, 1988: 1-403.

[11] Ren Fuyao, Complex Analytic Dynamic System, Shanghai China, Fudan University Press, 1996: 1-364.

[12] Shou Tiande, Neurobiology (2e), Higher Education Press, 2001, 2006: 1-548.

[13] Shou Tiande, Neurobiology, Higher Education Press, 2001, 2003: $1-470$.

[14] Su Jingcun, Topology of Manifold, Wuhan China, wuhan university press, 2005: 1-708.

[15] W. Miller, Symmetry Group and Its Application, Beijing China, Science Press, 1981: 1-486.

[16] Wu Chuanxi Li Guanghan, Submanifold geometry, Beijing China, Science Press, 2002: 1-217.

[17] Xiao Gang, Fibrosis of Algebraic Surfaces, Shanghai China, Shanghai science and technology Press, 1992: 1-180.

[18] Zhang Wenxiu Qiu Guofang, Uncertain Decision Making Based on Rough Sets, Beijing China, tsinghua university press, 2005: 1-255.

[19] Zheng jianhua, Meromorphic Functional Dynamics System, Beijing China, tsinghua university press, 2006: 1-413.

[20] Zheng Weiwei, Complex Variable Function and Integral Transform, Northwest Industrial University Press, 2011: 1-396.

[21] ЛаВрНТъеВ М. А., ІІІабаТ Б. В., Methods of Function of a Complex Variable Originally published in Russian under the title, 1956, 2006: 1-287.

[22] Zhu Rong Rong, Differential incremental equilibrium geometry protein granule Space folding, genome expression and cell modification, Fudan University, Volume 1, 2015-04-1: 1-112.

[23] Zhu Rong Rong, Differential incremental equilibrium geometry-protein granule Space folding, genome expression and cell modification General solution of nonlinear class of isolated wavelet -- effective nuclear trace information, Fudan University, Volume 2, 2015-04-11: 1-185.

[24] Zhu Rong Rong, Differential incremental equilibrium geometry - Effects of Cerebral Groove and Protein Granule Motion on Thinking Space and Mental Activity, 4-Dimensional Super-high-end Super-spherical Convex Spherical Fiber Cluster, Residual Product-like Cluster Petal Microfibers, Fudan University, Volume 3, 2015-04-18: 1-227. 